\title{
Magnons in ferromagnetic metallic manganites
}

\author{
Jiandi Zhang ${ }^{1}$, F Ye ${ }^{2}$, Hao Sha ${ }^{1}$, Pengcheng Dai ${ }^{2,3}$, J A Fernandez-Baca ${ }^{2}$ \\ and E W Plummer ${ }^{3,4}$ \\ ${ }^{1}$ Department of Physics, Florida International University, Miami, FL 33199, USA \\ ${ }^{2}$ Center for Neutron Scattering, Oak Ridge National Laboratory, Oak Ridge, TN 37831, USA \\ ${ }^{3}$ Department of Physics and Astronomy, The University of Tennessee, Knoxville, TN 37996, USA \\ ${ }^{4}$ Materials Science and Technology Division, Oak Ridge National Laboratory, Oak Ridge, \\ TN 37831, USA \\ E-mail: zhangj@fiu.edu
}

Received 16 January 2007, in final form 17 January 2007

Published 3 July 2007

Online at stacks.iop.org/JPhysCM/19/315204

\begin{abstract}
Ferromagnetic (FM) manganites, a group of likely half-metallic oxides, are of special interest not only because they are a testing ground for the classical double-exchange interaction mechanism for the 'colossal' magnetoresistance, but also because they exhibit an extraordinary arena of emergent phenomena. These emergent phenomena are related to the complexity associated with strong interplay between charge, spin, orbital, and lattice. In this review, we focus on the use of inelastic neutron scattering to study the spin dynamics, mainly the magnon excitations in this class of FM metallic materials. In particular, we discuss the unusual magnon softening and damping near the Brillouin zone boundary in relatively narrow-band compounds with strong Jahn-Teller lattice distortion and charge-orbital correlations. The anomalous behaviours of magnons in these compounds indicate the likelihood of cooperative excitations involving spin and lattice as well as orbital degrees of freedom.
\end{abstract}

(Some figures in this article are in colour only in the electronic version)

\section{Contents}

1. Introduction 2

2. Magnons in double exchange (DE) ferromagnets 3

3. Neutron as a probe for magnon excitations $\quad 5$

4. Magnons in high- $T_{\mathrm{C}}$ manganites $\quad 6$

5. Zone-boundary magnon softening $\quad 8$

6. Doping dependence of magnon excitations 11

7. Anomalous magnon damping 16

8. Temperature dependence and incoherent spin dynamics near $T_{\mathrm{C}} \quad 19$

9. Discussion of possible explanations 22

$\begin{array}{ll}\text { 10. Summary } & 25\end{array}$

0953-8984/07/315204+28\$30.00 C 2007 IOP Publishing Ltd Printed in the UK 1 


\section{Introduction}

Half-metallic ferromagnets are characterized by completely spin-polarized electronic density of states at the Fermi level, i.e., the majority spin channel is metallic while the Fermi energy falls in a band gap in the minority spin density of states [1]. In a class of doped manganites [2] which exhibit the colossal magnetoresistance (CMR) effect [3] - the extremely large drop in resistivity induced by application of a magnetic field near the Curie temperature $\left(T_{\mathrm{C}}\right)$, the FM metallic state has been suggested theoretically [4] and experimentally [5] as a possible halfmetallic state.

The revival in the study of manganites has led to the observation of a large array of emergent phase structures and transitions $[6,7]$. It is believed that the richness of physical properties results from the multitude of competing ground states-the equilibrium between phases is very subtle and small perturbations may induce a large response, which can be tuned by chemical doping, structural manipulation, strain induction, or the application of external stimuli, such as pressure, electric and magnetic fields, etc. In general, the fundamental physics behind these emergent phenomena is related to the complexity which is associated with strong interplay between charge, spin, orbital, and lattice.

The metallic ground state associated with FM order in doped manganites was originally understood by the DE interaction model [8]. In this model, the kinetics of itinerant electrons in these materials strongly correlates with localized spins in the Mn sites through the strong Hund's rule coupling. The electron hopping maintains its optimal nature when the net spins of $\mathrm{Mn}$ sites are all parallel. Consequently, FM ordering of the localized spins promotes a metallic state with high conductivity of electrons, and vice versa, while paramagnetic (PM) order prevents electrons from hopping and thus endorses an insulating state. Although the DE interaction has been recognized as a basic ingredient for the coupled FM metallic to $\mathrm{PM}$ insulator transition as well as the CMR effect, the nature of the FM metallic ground state is still not understood [6]. In particular, as we will focus on in this review, the spin dynamics in the FM metallic manganites is by no means conventional. The unconventional behaviours of spin dynamics in FM manganites are revealed by the deviation of the dispersion, the linewidth, and the long-wavelength stiffness of magnons from the expectations of the simple DE model. Based upon the fact that a strong interplay exists between different degrees of freedom and their excitations, several theoretical approaches beyond the simple DE model have been attempted. These include considering the magnon-phonon coupling, effects of electron-electron correlation, orbital fluctuations, and local phase inhomogeneities. Yet it is fair to conclude that none of the prevailing models can account for the observed magnon behaviours.

The paper is organized as follows. In section 2 we describe the magnons in a canonical DE FM system with a strong Hund's rule coupling including expected magnon dispersion, lifetime, and stiffness. Section 3 contains a brief description of inelastic neutron scattering (INS) as an ideal probe to measure the magnon properties. The results of magnon measurements from relatively high- $T_{\mathrm{C}}$ or large-bandwidth manganites are reviewed in section 4 . Sections 5 and 6 present the results of magnon measurements from low- $T_{\mathrm{C}}$ compounds with strong Jahn-Teller (JT) and other correlation effects where the unusual magnon behaviours were observed. In section 7 we discuss magnon damping and possible correlation with lattice dynamics. Section 8 reports results on the incoherent spin dynamics when temperature approaches to $T_{\mathrm{C}}$ and the possible correlations with phase separation. Some theoretical approaches that account for these 


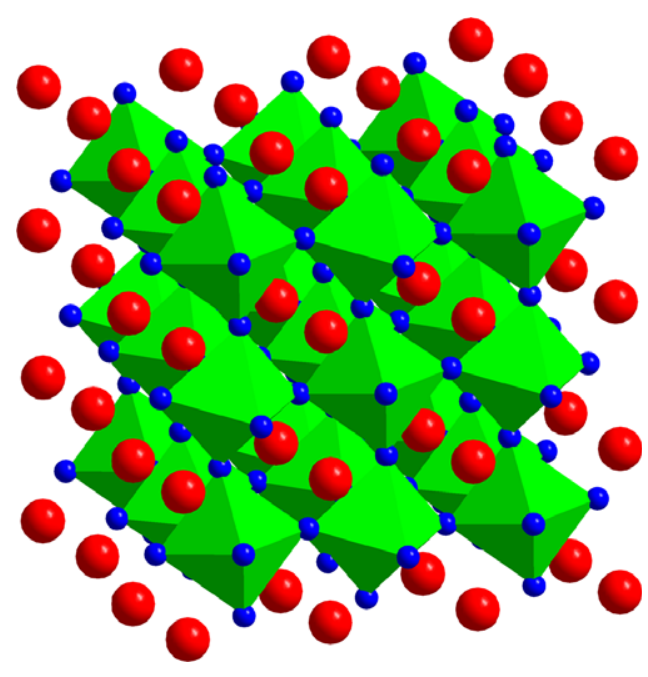

Figure 1. Ball model of the crystal structure of typical FM metallic $\mathrm{R}_{1-x} \mathrm{~A}_{x} \mathrm{MnO}_{3}$ manganites with pseudocubic perovskite (orthorhombic) symmetry. Big (red) balls represent cation elements, small (blue) ones are oxygen ions, while $\mathrm{Mn}$ ions are in the centres of green octahedron cages.

observed magnon behaviours, especially the zone boundary magnon softening, are discussed in section 9. A brief summary is given in section 10 .

\section{Magnons in double exchange (DE) ferromagnets}

In this review, we concentrate on perovskite manganites with a transition from a hightemperature $\mathrm{PM}$ insulator to a low-temperature $\mathrm{FM}$ metal at $T_{\mathrm{C}}$, mainly pseudo-cubic perovskite manganites $\mathrm{R}_{1-x} \mathrm{~A}_{x} \mathrm{MnO}_{3}$ (e.g. $\mathrm{R}=\mathrm{La}, \mathrm{Nd}, \mathrm{Pr}, \mathrm{A}=\mathrm{Sr}, \mathrm{Ca}, \mathrm{Pb}$ ) (see figure 1). The compounds that exhibit this behaviour have been partially hole-doped away from a parent antiferromagnetic (AF) insulator $\mathrm{RMnO}_{3}$ by divalent substitution on the cation site, such as $\mathrm{La}_{0.7} \mathrm{Ca}_{0.3} \mathrm{MnO}_{3}$. The Mn $3 \mathrm{~d}$ levels, split by the oxygen octahedral crystal field to a lowerenergy $t_{2 g}$ triplet and a higher-energy $e_{g}$ doublet, are filled according to Hund's rule such that all spins are aligned on a given site by a large intra-atomic exchange $J_{\mathrm{H}}$. Electronic conduction arises from the hopping of an electron from $\mathrm{Mn}^{3+}$ to $\mathrm{Mn}^{4+}$ with electron transfer energy $t$. In general, these systems can be treated as a single $e_{\mathrm{g}}$ band of electrons interacting with localized core spins in $\mathrm{t}_{2 \mathrm{~g}}$ triplet by a Hund rule exchange interaction and described by Kondo-type lattice model [9-11]:

$$
H=-t \sum_{\langle i, j\rangle, \alpha}\left(c_{i \alpha}^{+} c_{j \alpha}+c_{j \alpha}^{+} c_{i \alpha}\right)-\frac{J_{\mathrm{H}}}{2 S} \sum_{i, \alpha, \beta} \vec{S}_{i} \cdot \vec{\sigma}^{\alpha \beta} c_{i \alpha}^{+} c_{i \beta}
$$

where $c_{j \sigma}$ is the fermionic operator corresponding to conduction electrons, hopping between the atomic sites of magnetic Mn ions with spins $\vec{S}_{i}(S=3 / 2)$, and the vector $\vec{\sigma}^{\alpha \beta}$ is composed of Pauli matrices. In the limit of $J_{\mathrm{H}} \gg t$, the itinerant conduction electrons must be locally aligned with the core spins on any site such that the ground state is an FM state. The ferromagnetic interaction between core spins mediated by conduction electrons is referred as the DE model [8]. In the canonical limit $t / J_{\mathrm{H}} \rightarrow 0$ and large- $S$ approximation [12], the Kondotype model is equivalent to the nearest-neighbour Heisenberg ferromagnet, which is generally described by

$$
H=-\sum_{i j} J_{i j} \vec{S}_{i} \cdot \vec{S}_{j}
$$




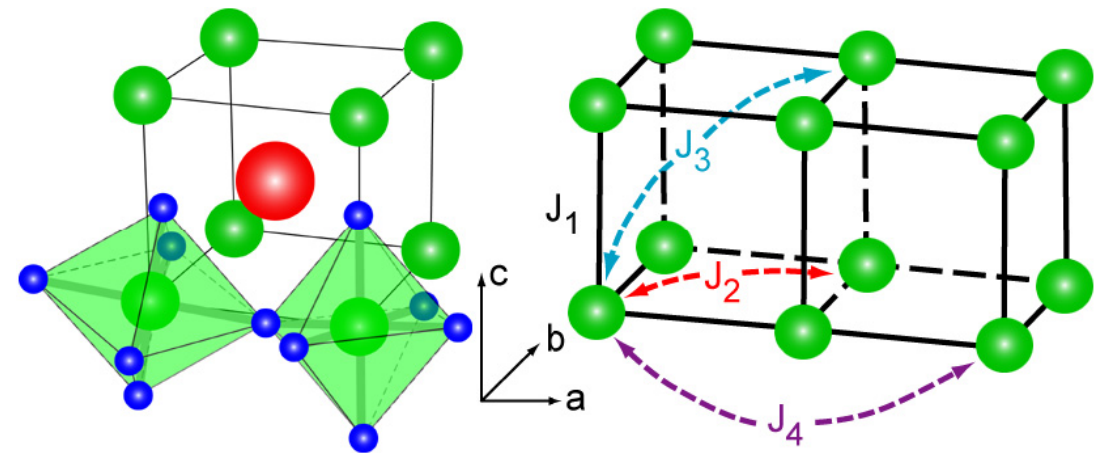

Figure 2. Pseudo-cubic crystal structure of $\mathrm{R}_{1-x} \mathrm{~A}_{x} \mathrm{MnO}_{3}$ with different $\mathrm{Mn}$ neighbours of magnetic exchange coupling indicated. The biggest (red) ball represents cation elements, the small (blue) ones oxygen ions, and the medium size (green) ones Mn ions.

with coupling $J_{i j}$ between pairs of spins at sites $\vec{R}_{i}$ and $\vec{R}_{j}$. For an FM ground state and in a linear approximation, the corresponding magnon dispersion is given by

$$
\hbar \omega(\vec{q})=\Delta+2 S[J(\overrightarrow{0})-J(\vec{q})]
$$

where $\vec{q}$ is the momentum transfer (or momentum transfer in the first Brillouin zone or reduced vector) during the magnon excitations, $\Delta$ is the magnon energy gap representing the energy to uniformly rotate the entire spin system away from the easy direction of magnetization (thus sometimes called the magnetic anisotropy gap) and

$$
J(\vec{q})=\sum_{j} J_{i j} \exp \left[\mathrm{i} \vec{q} \cdot\left(\vec{R}_{i}-\vec{R}_{j}\right)\right]
$$

for a Bravais lattice. For a pseudo-cubic crystal structure of $\mathrm{R}_{1-x} \mathrm{~A}_{x} \mathrm{MnO}_{3}$, the first few neighbour exchanging couplings are schematically shown in figure 2 . In addition to the nearestneighbour interaction with exchange coupling constant $J_{1}$ along the equivalent $[1,0,0],[0,1$, $0]$, or $[0,0,1]$ direction, $J_{2}$ represents the next-nearest-neighbour exchange coupling, thus is the coupling in the planar $[1,1,0]$ direction. $J_{3}$ is the coupling in the cubic diagonal $[1,1$, 1] direction. $J_{4}$ is the next-neighbour coupling along the same directions as $J_{1}$. In the longwavelength limit $\vec{q} \rightarrow 0$, equation (3) reduces to

$$
\hbar \omega(\vec{q})=\Delta+D q^{2}
$$

in which

$$
D=\frac{S}{3} \sum_{j} J_{i j}\left|\vec{R}_{i}-\vec{R}_{j}\right|^{2}
$$

is defined as the spin stiffness for a pseudo-cubic system. For the simplest case where only the nearest-neighbour coupling is considered, the magnon dispersion equation (3) further reduces to $[10]$

$$
\hbar \omega(\vec{q}) \cong \Delta+4 J_{1} S\left[3-\cos \left(q_{x} a_{0}\right)-\cos \left(q_{y} a_{0}\right)-\cos \left(q_{z} a_{0}\right)\right]
$$

where $J_{1}$ is the nearest-neighbour exchange constant. Here we define $\left(q_{x}, q_{y}, q_{z}\right) \equiv$ $\left(\frac{2 \pi}{a_{0}} h, \frac{2 \pi}{a_{0}} k, \frac{2 \pi}{a_{0}} l\right)$. For a cubic system, the spin stiffness shown in equation (6) can be further simplified as

$$
D=8 \pi^{2} S J_{1}
$$


in the reciprocal-lattice units (rlu). In the mean filed Heisenberg model [13], the Curie temperature is directly proportional to the exchange constant [12],

$$
T_{\mathrm{C}}=\frac{4 S(S+1)}{k_{\mathrm{B}}} J_{1} \text {. }
$$

It should be noted that, in practice, any quantum fluctuation effect tends to reduce the effective $T_{\mathrm{C}}$ even in the Heisenberg ferromagnet. The magnon bandwidth is also determined by

$$
W_{\mathrm{sw}} \equiv \hbar \omega\left[\vec{q}=\left(\frac{1}{2}, \frac{1}{2}, \frac{1}{2}\right)\right]=24 S J_{1} .
$$

Under this scenario, the spin-wave stiffness $D$ and bandwidth should be linearly proportional to the Curie temperature $\left(T_{\mathrm{C}}\right)$. In a mean-field theory for a Heisenberg FM case, $T_{\mathrm{C}}$ like $J_{1}$ is proportional to the average kinetic energy ( $t$ ) [9-12], i.e., $D \propto J_{1} \propto t \propto T_{\mathrm{C}}$.

In this simple Heisenberg ferromagnet, the spin waves are the exact eigenstates of the Heisenberg Hamiltonian. At $T=0 \mathrm{~K}$, magnons are non-interacting quasiparticles with long lifetime and no damping.

\section{Neutron as a probe for magnon excitations}

Neutron scattering has been a vital tool in probing both magnetic ordering and spin dynamics such as magnon excitations $[14,15]$. For unpolarized inelastic scattering neutrons with momentum transfer vector $\vec{Q}=\vec{k}_{i}-\vec{k}_{f}=\vec{G}+\vec{q}$, where $\vec{G}$ is the reciprocal-lattice vector and energy transfer $\hbar \omega=\frac{\hbar^{2}}{2 m_{n}}\left(k_{i}^{2}-k_{f}^{2}\right)$, the differential cross section for the scattering from a system of electron spins is given by

$$
\frac{\mathrm{d}^{2} \sigma}{\mathrm{d} \Omega_{f} \mathrm{~d} E_{f}}=\frac{N}{\hbar} \frac{k_{f}}{k_{i}} p^{2} \mathrm{e}^{-2 W} \sum_{\alpha \beta}\left(\delta_{\alpha \beta}-\hat{Q}_{\alpha} \hat{Q}_{\beta}\right) S^{\alpha \beta}(\vec{Q}, \omega)
$$

with the scattering function

$$
S^{\alpha \beta}(\vec{Q}, \omega)=\frac{1}{2 \pi} \int_{-\infty}^{\infty} \mathrm{d} t \mathrm{e}^{-\mathrm{i} \omega t} \sum_{l}\left\langle S_{0}^{\alpha}(0) S_{l}^{\beta}(t)\right\rangle
$$

where $\langle\cdots\rangle$ denotes an average over configurations. For magnetic ions, the amplitude for magnetic scattering is given by $p=\left(\frac{\gamma r_{0}}{2}\right) g f(\vec{Q})$ where $\gamma=1.913$ is the neutron gyromagnetic ratio, $r_{0}$ the classical electron radius, and $f(\vec{Q})=\int \rho_{\mathrm{s}}(\vec{r}) \mathrm{e}^{\mathrm{i} \vec{Q} \cdot \vec{r}} \mathrm{~d} r$ the magnetic form factor which is the Fourier transform of the normalized unpolarized spin density $\rho_{\mathrm{s}}(\vec{r})$ on an atom. For magnon scattering, a neutron, scattering from a magnetic system, can adsorb or emit one or more magnons. For a single magnon process with collinear spins aligned parallel to the $z$-axis such that the magnons involve $S^{x}$ and $S^{y}$, then summation in equation (11) can be expressed as

$$
\sum_{\alpha \beta}\left(\delta_{\alpha \beta}-\hat{Q}_{\alpha} \hat{Q}_{\beta}\right) S^{\alpha \beta}(\vec{Q}, \omega)=\frac{1}{2}\left(1+\hat{Q}_{z}^{2}\right) S_{\mathrm{sw}}(\vec{Q}, \omega)
$$

where $S_{\mathrm{sw}}(\vec{Q}, \omega)$ is the inelastic scattering function for magnons. In particular, for a simple Heisenberg ferromagnet with small $\vec{q}=\vec{Q}-\vec{G}$ where magnons exhibit parabolic-type dispersion, the inelastic scattering function can be further simplified as

$S_{\mathrm{sw}}(\vec{Q}, \omega)=S \sum_{\vec{G}, \vec{q}}\left[\left(n_{\vec{q}}+1\right) \delta(\vec{Q}-\vec{q}-\vec{G}) \delta\left(\omega-\omega_{\vec{q}}\right)+n_{\vec{q}} \delta(\vec{Q}+\vec{q}-\vec{G}) \delta\left(\omega+\omega_{\vec{q}}\right)\right]$.

The essential information contained in neutron scattering is that neutrons scattered with momentum and energy transfer $\vec{Q}$ and $\hbar \omega$ directly probe a single Fourier component of the spin-pair correlation function (i.e. the scattering function). For a given energy, the scattering 
function as a function of $\vec{Q}$ provides information on the dynamic spin-spin correlations. In reality, magnon-magnon interaction and other channels of interactions (like magnon-phonon) lead to magnon damping [15]. To include the effect of damping it is convenient to make use of the fluctuation-dissipation theorem to relate the scattering function to the imaginary part of the generalized susceptibility $[15,16]$ :

$$
S^{\alpha \beta}(\vec{Q}, \omega)=[n(\omega)+1] \operatorname{Im} \chi^{\alpha \beta}(\vec{Q}, \omega)
$$

where $n(\omega)+1=\left[1-\exp \left(-\frac{\hbar \omega}{k_{\mathrm{B}} T}\right)\right]^{-1}$ is the Bose population factor. Therefore, the neutron scattering directly measures $\operatorname{Im} \chi^{\alpha \beta}(\vec{Q}, \omega)$, hence providing information on magnon damping due to the existence of different interactions. For example, in the damped simple harmonic oscillator (DSHO) approximation [16], the normalized dynamic susceptibility $\operatorname{Im} \chi^{\alpha \beta}(\vec{Q}, \omega)$ can be expressed as

$$
\operatorname{Im} \chi(\vec{Q}, \omega)=\frac{4 \gamma \omega \omega_{0}}{\pi\left[\left(\omega^{2}-\omega_{0}^{2}\right)^{2}+4(\gamma \omega)^{2}\right.}
$$

where $\gamma$ characterizes the magnon damping while $\omega_{0}$ is associated with the magnon dispersion relation.

The experimental results presented below were mostly obtained from the inelastic neutron scattering of single-crystal manganites. Most of the experiments were performed on tripleaxis neutron scattering spectrometers except where otherwise indicated. The manganite crystals for the experiments were mainly grown by the travelling solvent floating zone technique. The reciprocal-lattice units (rlu) are used to label wavevectors so that the momentum transfers $\left(q_{x}, q_{y}, q_{z}\right)$ in units of $\AA^{-1}$ are at reciprocal space positions $(H, K, L)=$ $\left(q_{x} a_{x} / 2 \pi, q_{y} a_{y} / 2 \pi, q_{z} a_{z} / 2 \pi\right)$ rlu, where $a_{x}, a_{y}$, and $a_{z}$ are the lattice parameters. For simplicity, we label all wavevectors in terms of the pseudo-cubic unit cells with lattice parameter $a$. In reality, most manganites have a lower-symmetry structure such as an orthorhombic one, which is slightly distorted from the cubic lattice (see the ball structure model for an orthorhombic phase in figure 1). In the pseudocubic perovskite unit cell, $a_{x}=a_{y}=a_{z}=a$. In this notation, the zone boundaries along the $[\xi, 0,0],[\xi, \xi, 0]$, and $[\xi, \xi, \xi]$ directions for FM magnons are at $(0.5,0,0) \mathrm{rlu},(0.5,0.5,0) \mathrm{rlu}$, and $(0.5,0.5,0.5) \mathrm{rlu}$, respectively.

\section{Magnons in high- $T_{\mathrm{C}}$ manganites}

The earlier experiments on the measurement of magnon excitations were carried out from the crystals of $\mathrm{La}_{0.7} \mathrm{~Pb}_{0.3} \mathrm{MnO}_{3}$ by Perring et al [17], $\mathrm{La}_{0.7} \mathrm{Sr}_{0.3} \mathrm{MnO}_{3}$ by Martin et al [18] and $\mathrm{La}_{0.8} \mathrm{Sr}_{0.2} \mathrm{MnO}_{3}$ by Endoh et al [19]. For these compounds with higher Curie temperature $\left(T_{\mathrm{C}}=355 \mathrm{~K}\right.$ for $\mathrm{La}_{0.7} \mathrm{~Pb}_{0.3} \mathrm{MnO}_{3}, 378 \mathrm{~K}$ for $\mathrm{La}_{0.7} \mathrm{Sr}_{0.3} \mathrm{MnO}_{3}$, and $312 \mathrm{~K}$ for $\left.\mathrm{La}_{0.8} \mathrm{Sr}_{0.2} \mathrm{MnO}_{3}\right)$, the dispersion of magnons along all three high-symmetry directions, [1, 0, 0], [1, 1, 0], and $[1,1,1]$, was explained by a simple Heisenberg model with solely a nearest-neighbour coupling. As shown in figure 3, the dispersion relation using equation (7) is entirely sufficient to account for the data obtained from $\mathrm{La}_{0.7} \mathrm{~Pb}_{0.3} \mathrm{MnO}_{3}$ at $10 \mathrm{~K}$ [17], giving the spin-wave stiffness $D \cong 133.7 \mathrm{meV} \AA^{2}$ with $2 J_{1} S=8.79 \pm 0.21 \mathrm{meV}$ and $\Delta=2.51 \pm 0.46 \mathrm{meV}$. The value of the gap $\Delta$ was obtained by fitting the dispersion data to the model instead of that from direct measurement. It was found that adding the second- and third-nearest-neighbour exchange interactions does not improve the fit. The simple nearest-neighbour FM Heisenberg model, with a consideration of the effect of fluctuations [13], also accounts for the estimate of $T_{\mathrm{C}}$ of the materials to within $15 \%$. On the other hand, the data for the low-energy and 


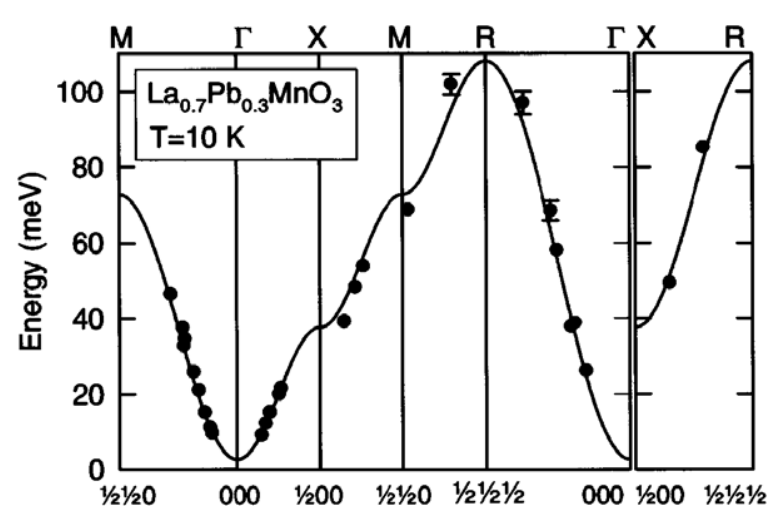

Figure 3. Magnon dispersion of $\mathrm{La}_{0.7} \mathrm{~Pb}_{0.3} \mathrm{MnO}_{3}$ along the three major cubic symmetry directions at $10 \mathrm{~K}$ determined by inelastic neutron scattering. Solid lines are the fit to the Heisenberg model with nearest-neighbour coupling $2 J_{1} S=8.79 \pm 0.21 \mathrm{meV}$. The figure is taken from [17].

near-zone-boundary magnon excitations are still lacking. A more complete data set to map the magnon dispersion and extract the value of spin-wave stiffness $D$ is desirable.

The results of the magnon dispersion at long wavelength for $\mathrm{La}_{0.7} \mathrm{Sr}_{0.3} \mathrm{MnO}_{3}$ along the $[1,1,0]$ direction measured by Martin et al [18] also suggested that the dispersion can be understood by the simple Heisenberg model. The fit to the data obtained at $10 \mathrm{~K}$ for the dispersion gave the spin-wave stiffness $D=188 \pm 8 \mathrm{meV} \AA^{2}$ and a very small fitting gap $\Delta=0.75 \pm 0.40 \mathrm{meV}$. Vasiliu-Doloc et al [19] obtained $D=176 \pm 5 \mathrm{meV} \AA^{2}$ for $\mathrm{La}_{0.7} \mathrm{Sr}_{0.3} \mathrm{MnO}_{3}$ and $166.8 \pm 5 \mathrm{meV} \AA^{2}$ for $\mathrm{La}_{0.8} \mathrm{Sr}_{0.2} \mathrm{MnO}_{3}$ at $15 \mathrm{~K}$, although the earlier and less accurate measurements [20] gave a lower value of $D$. However, no energy gap (at least $\Delta<0.02 \mathrm{meV}$, which is within the instrumental energy resolution) was measured. From this value of $D$ one would be able to calculate the mean-field value of $T_{\mathrm{C}}$ based upon equations (8) and (9). It was found [18] that the calculated $T_{\mathrm{C}}$ value is more than twice as high as the actual $T_{\mathrm{C}}=378 \mathrm{~K}$ of the system. This has been used as an indication for the itinerant character of the system, since an itinerant ferromagnet generally has a lower $T_{\mathrm{C}}$ compared to the meanfield $T_{\mathrm{C}}$ value but large $D$ value [20]. Obviously this argument assumes that magnons follow completely the cosine-like dispersion (equation (7)) such that a large $D$ value would have a large magnon bandwidth. Unfortunately, the magnon dispersion was mapped only in the low- $q$ range, while the exact magnon bandwidth was not clear for this system. A complete magnon dispersion to the zone boundary along the $[1,0,0]$ direction has recently been reported [21] but a zone-boundary softening deviating from the nearest-neighbour Heisenberg model has been observed. We will discuss this in the next section.

The experimental results on the magnon dispersion and the doping $(x)$ dependence of spinwave stiffness for $\mathrm{La}_{1-x} \mathrm{Sr}_{x} \mathrm{MnO}_{3}(x \leqslant 0.3)$ seem to further confirm the validity of the nearestneighbour Heisenberg model. Based upon the long-wavelength part of the magnon dispersion, Endoh and Hirota [20] discovered that the $x$ dependence of $D$ almost completely coincided with that of $T_{\mathrm{C}}$, both solely depending on $J_{1}$, thus following the simple relationship $D \propto J_{1} \propto T_{\mathrm{C}}$ as predicted by the nearest-neighbour Heisenberg model. Figure 4 summarizes the correlation between measured transition temperature (either the Curie or Néel temperature [22]) and spinwave stiffness as a function of doping concentration of $\mathrm{La}_{1-x} \mathrm{Sr}_{x} \mathrm{MnO}_{3}$ from those reported by [20] and other measurements $[18,19,23]$. Moreover, the ratio between the calculated $T_{\mathrm{C}}$ based upon equation (9) by using the experimentally determined $J_{1}$ and the actual $T_{\mathrm{C}}$ is almost the same in the doping range studied $(x \leqslant 0.3)$. However, it is known that there is an FM 


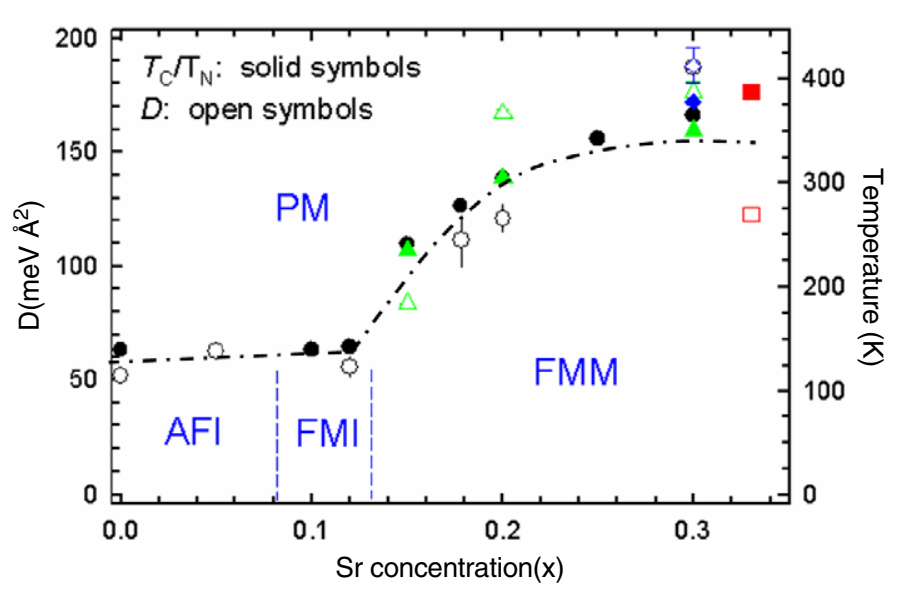

Figure 4. Sr-doping dependence of $T_{\mathrm{C}} / T_{\mathrm{N}}$ and spin-wave stiffness $D$ in $\mathrm{La}_{1-x} \mathrm{Sr}_{x} \mathrm{MnO}_{3}$. The dashed lines are guides to the eye. The figure follows the plot of figure 1 in [20] but new data points are added.

metal to FM insulator transition below $x=0.175$. Thus from the independence of the ratio from doping it has been speculated that the electron correlation energy remains essentially unchanged across the metal-to-insulator transition of $\mathrm{La}_{1-x} \mathrm{Sr}_{x} \mathrm{MnO}_{3}$.

\section{Zone-boundary magnon softening}

Evidence of the magnon behaviour deviating from the simple nearest-neighbour Heisenberg model was first discovered in $\mathrm{Pr}_{0.63} \mathrm{Sr}_{0.37} \mathrm{MnO}_{3}$ with $T_{\mathrm{C}}=301 \mathrm{~K}$ [24]. A clear softening of the magnon dispersion at the zone boundary for $T<T_{\mathrm{C}}$ and significant broadening of the zone boundary magnons as $T \rightarrow T_{\mathrm{C}}$ have been observed. Figure 5 shows the magnon dispersion of $\operatorname{Pr}_{0.63} \mathrm{Sr}_{0.37} \mathrm{MnO}_{3}$ along the three high-symmetry directions. It can be seen that only the nearest-neighbour coupling is not enough to account for the dispersion of magnon. The solid line in figure 5 is the result of a fit to only nearest-neighbour interactions for the small- $q$ range $(\xi<0.2)$, resulting in $\Delta=1.3 \pm 0.3 \mathrm{meV}$ and $2 J_{1} S=8.2 \pm 0.5 \mathrm{meV}$. Though fitted results for the small- $q$ range are similar to those obtained from the dispersion in $\mathrm{La}_{0.7} \mathrm{~Pb}_{0.3} \mathrm{MnO}_{3}$, a large deviation by $15-20 \mathrm{meV}$ near the zone boundary ( $\xi=0.5$ in cubic structure) is evident, in sharp contrast with that in $\mathrm{La}_{0.7} \mathrm{~Pb}_{0.3} \mathrm{MnO}_{3}$. The spin-wave stiffness $D$ of $\operatorname{Pr}_{0.63} \mathrm{Sr}_{0.37} \mathrm{MnO}_{3}$ is $165 \mathrm{meV} \AA^{2}[24]$.

A Heisenberg model including higher-order couplings to fourth-neighbour interactions has been taken to fit the full data set and the results are shown in figure 5 (dashed curves) [24]. This fit gives $\Delta=0.2 \pm 0.3 \mathrm{meV}, 2 J_{1} S=5.58 \pm 0.07 \mathrm{meV}, 2 J_{2} S=-0.36 \pm 0.04 \mathrm{meV}$, $2 J_{3} S=0.36 \pm 0.04 \mathrm{meV}$, and $2 J_{4} S=1.48 \pm 0.10 \mathrm{meV}$. It was also found that a better fit for data near the zone boundary in the $[0,0,1]$ direction required the next Fourier term $\left(J_{8}\right)$ as compared with that in the $[1,1,0]$ and $[1,1,1]$ directions. Though $J_{2}$ and $J_{3}$ were necessary to fit the data, the fourth-nearest-neighbour coupling $J_{4}$ is particularly important to correct the nearest-neighbour coupling. It seems that the long-range and non-monotonic behaviour of $J(\vec{q})$ required by the measured data rules out a simple Heisenberg Hamiltonian with nearestneighbour exchange coupling.

Such a strong zone-boundary magnon softening has been further confirmed by other measurements on several manganites with relatively low $T_{\mathrm{C}}$ [25-28]. Compared with 


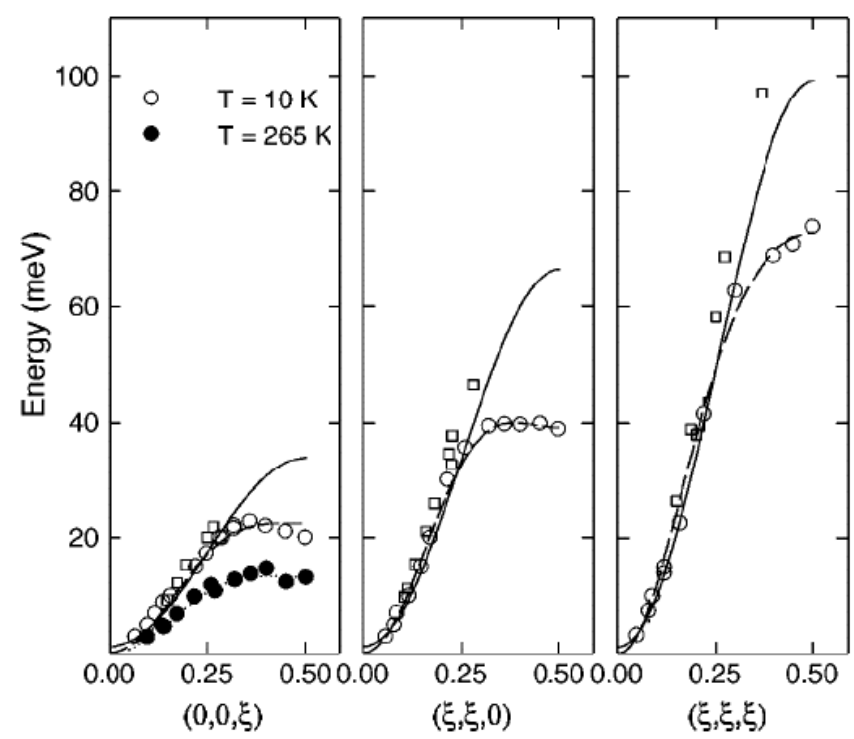

Figure 5. Magnon dispersion of $\operatorname{Pr}_{0.63} \mathrm{Sr}_{0.37} \mathrm{MnO}_{3}$ along the $[0,0,1],[1,1,0]$, and $[1,1,1]$ directions (the zone boundary is at $\xi=0.5$ ) [24]. The solid line is a fit to a nearest-neighbour Heisenberg Hamiltonian for $T=10 \mathrm{~K}$ and $\xi<0.2$ while the dashed curve is a fit for all data including up to the fourth-nearest-neighbour couplings at $T=10 \mathrm{~K}$. The dotted line is the corresponding fit for $T=265 \mathrm{~K}$. Also shown in squares are the data for $\mathrm{La}_{0.7} \mathrm{~Pb}_{0.3} \mathrm{MnO}_{3}$ (see figure 2 and [17]).

$\operatorname{Pr}_{0.63} \mathrm{Sr}_{0.37} \mathrm{MnO}_{3}\left(T_{\mathrm{C}}=301 \mathrm{~K}\right), \mathrm{La}_{0.7} \mathrm{Ca}_{0.3} \mathrm{MnO}_{3}\left(T_{\mathrm{C}}=238 \mathrm{~K}\right)$ and $\mathrm{Nd}_{0.7} \mathrm{Sr}_{0.3} \mathrm{MnO}_{3}$ $\left(T_{\mathrm{C}}=198 \mathrm{~K}\right)$ have much lower $T_{\mathrm{C}}$, though all three compounds have an FM metallic ground state, have an identical $T$ dependence of resistivity, and exhibit a metal-to-insulator transition around $T_{\mathrm{C}}$ (see figure 6). In particular, $\mathrm{La}_{0.7} \mathrm{Ca}_{0.3} \mathrm{MnO}_{3}$ is a widely studied manganite with the optimized doping level for the CMR effect [29]. One advantage of using $\mathrm{La}_{0.7} \mathrm{Ca}_{0.3} \mathrm{MnO}_{3}$ to study the magnon behaviour is that $\mathrm{La}$ is not a magnetic ion so that no excitation due to the crystal electric field (CEF) level is involved in magnon excitations. Dai et al [27] have carried out detailed studies on the magnon dispersion and damping, as well as its temperature dependence. Figure 7 presents a complete set of constant- $\boldsymbol{q}$ scans in the $[1,0,0]$ direction (the same as the $[0,0,1]$ direction in the notation for cubic perovskite structure through this paper) for the magnon excitations of $\mathrm{La}_{0.7} \mathrm{Ca}_{0.3} \mathrm{MnO}_{3}$ at $10 \mathrm{~K}$. The magnon peaks are well resolved up to the zone boundary without any other magnetic excitations (like those due to CEF) in the observed energy window. However, a large increase in linewidth and decrease in intensity near the zone boundary are evident in the magnon excitation spectra. Figure 8 presents the magnon dispersions of $\mathrm{La}_{0.7} \mathrm{Ca}_{0.3} \mathrm{MnO}_{3}$ accompanied by $\mathrm{Nd}_{0.7} \mathrm{Sr}_{0.3} \mathrm{MnO}_{3}$ and $\mathrm{Pr}_{0.63} \mathrm{Sr}_{0.37} \mathrm{MnO}_{3}$ along both $[0,0,1]$ and $[1,1,0]$ directions. Remarkably, the magnon dispersions of these three manganites are almost identical at the measured energies, showing a large zone boundary softening in both $[0,0,1]$ and $[1,1,0]$ directions. This indicates that the magnetic exchange coupling strength is insensitive to large difference (more than $100 \mathrm{~K}$ ) of $T_{\mathrm{C}}$ values, in sharp contrast to the prediction of $D \propto J_{1} \propto t \propto T_{\mathrm{C}}$. These are conclusive evidence that the spin dynamics in these manganites cannot be explained by a simple nearest-neighbour Heisenberg model.

Endoh et al [28] have measured the magnon excitation of the FM state of $\mathrm{Sm}_{0.55} \mathrm{Sr}_{0.45} \mathrm{MnO}_{3}$ which has much lower $T_{\mathrm{C}}(\sim 135 \mathrm{~K})$ and is located on the verge of a doping- 


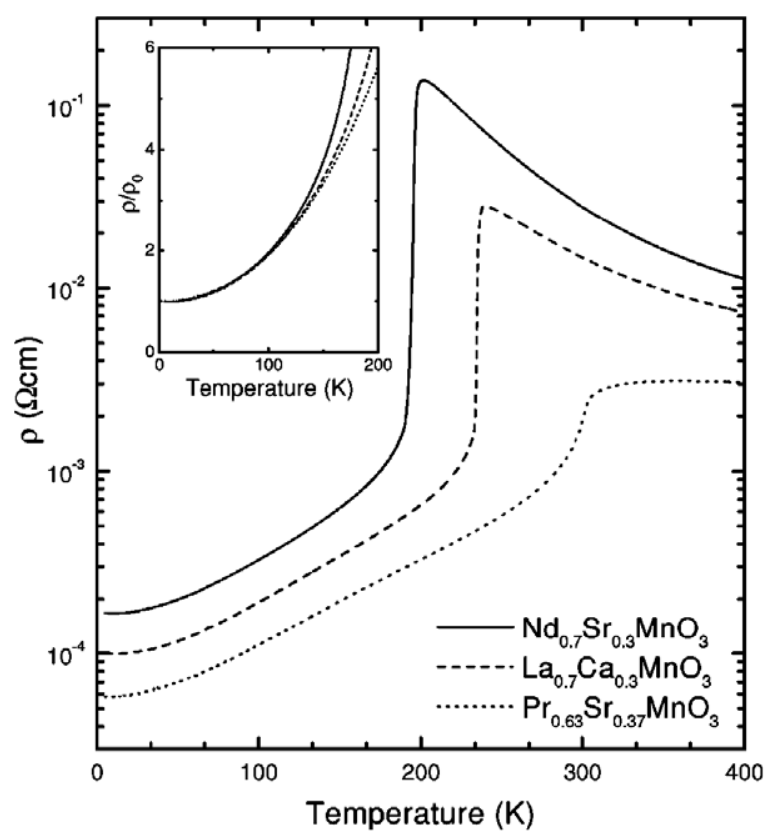

Figure 6. $T$ dependence of the resistivity $\rho(T)$ of the single crystal $\mathrm{Nd}_{0.7} \mathrm{Sr}_{0.37} \mathrm{MnO}_{3}$, $\mathrm{La}_{0.7} \mathrm{Ca}_{0.3} \mathrm{MnO}_{3}$, and $\operatorname{Pr}_{0.63} \mathrm{Ca}_{0.37} \mathrm{MnO}_{3}$ for the magnon measurements [27]. The large drop in $\rho(T)$ corresponds to the $T_{\mathrm{C}}$ at 198,238 , and $301 \mathrm{k}$, respectively. The inset shows the normalized resistivity $\rho(T) / \rho(0)$. Note that all three compounds have the same $T$ dependence of $\rho(T) / \rho(0)$ in the FM metallic state below $100 \mathrm{~K}$.

induced metal-insulator transition [30]. The anomalous zone-boundary magnon softening has also been observed in this low- $T_{\mathrm{C}}$ material along the $[1,0,0],[1,1,0]$ and $[1,1,1]$ directions. In particular, an anisotropic softening was observed with the largest softening in the $[1,0,0]$ direction. Yet, no obvious broadening of the magnon spectra has been observed near the zone boundary, where the dispersion tends to show softening, in sharp contrast to those observed in $\mathrm{Pr}_{0.63} \mathrm{Sr}_{0.37} \mathrm{MnO}_{3}, \mathrm{La}_{0.7} \mathrm{Ca}_{0.3} \mathrm{MnO}_{3}$, and $\mathrm{Nd}_{0.7} \mathrm{Sr}_{0.3} \mathrm{MnO}_{3}$. The magnon dispersions of $\mathrm{Sm}_{0.55} \mathrm{Sr}_{0.45} \mathrm{MnO}_{3}$ have been fitted to the Heisenberg model with the nearest-neighbour $\left(J_{1}\right)$ and fourth-neighbour $\left(J_{4}\right)$ couplings. However, the fits for the zone boundary dispersion along the $[1,1,0]$ and $[1,1,1]$ directions are not as good as that along the [1, 0, 0] direction [28], thus giving possible uncertainty in the determination of $J_{4}$. Nevertheless, it is found that the ratio $J_{4} / J_{1}(\sim 0.6)$ is much larger than those obtained from other compounds (see table 1$)$.

In contrast with the results for high- $T_{\mathrm{C}}$ manganites as we discussed in the previous section, the zone boundary softening has been reported in $\mathrm{La}_{0.68} \mathrm{Ba}_{0.32} \mathrm{MnO}_{3}$ [31] and $\mathrm{La}_{0.7} \mathrm{Ba}_{0.3} \mathrm{MnO}_{3}$ [32], which also have relatively high $T_{\mathrm{C}}$. A fit of the reported data [31] for $\mathrm{La}_{0.68} \mathrm{Ba}_{0.32} \mathrm{MnO}_{3}\left(T_{\mathrm{C}}=336 \mathrm{~K}\right)$ indicates a non-zero fourth-neighbour coupling with $2 J_{4}=1.59 \mathrm{meV}$ (see table 1). Chatterji et al [32] have measured the magnon dispersions of $\mathrm{La}_{0.7} \mathrm{Ba}_{0.3} \mathrm{MnO}_{3}\left(T_{\mathrm{C}}=350 \mathrm{~K}\right)$ at $1.5 \mathrm{~K}$ along the $[1,0,0]$ and $[1,1,0]$ directions and determined the spin-wave stiffness $D=152 \pm 3 \mathrm{meV} \AA^{2}$ by fitting the data to the Heisenberg model. However, a large deviation of the fitting curves from the experimental dispersions was found near the zone boundary. The magnons show zone boundary softening and are heavily damped for higher $q$ with larger linewidths than the instrumental resolution. In order to fit the dispersion data in the whole $q$ range, higher-order terms in the Heisenberg model need to be 


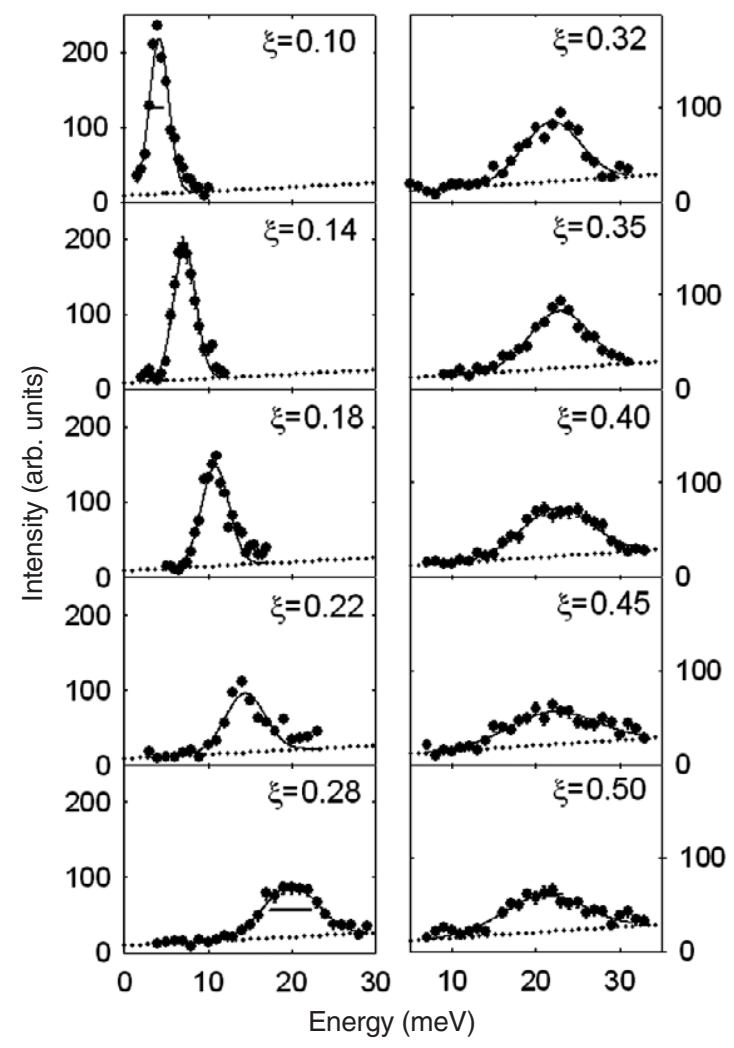

Figure 7. Sequence of the constant- $\boldsymbol{q}$ scans at selected wavevectors for the magnon excitations in $\mathrm{La}_{0.7} \mathrm{Ca}_{0.3} \mathrm{MnO}_{3}$ along the $[0,0,1]$ direction at $T=10 \mathrm{~K}$. The solid curves are the Gaussian fits to the data and the dashed lines represent the linear backgrounds.

taken into account (see table 1), in contrast with those obtained from $\mathrm{La}_{0.7} \mathrm{~Pb}_{0.3} \mathrm{MnO}_{3}$ [17]. So far it is still an open issue whether or not the zone-boundary magnon softening and damping are generic features of all FM manganites. As we have mentioned above, more measurements are needed on $\mathrm{La}_{0.7} \mathrm{~Pb}_{0.3} \mathrm{MnO}_{3}$ to determine the entire dispersion curve. There may not be enough data at present to conclude that the magnon behaviour in $\mathrm{La}_{0.7} \mathrm{~Pb}_{0.3} \mathrm{MnO}_{3}$ or other high- $T_{\mathrm{C}}$ manganites can indeed be described by a simple Heisenberg model with nearest-neighbour exchange interaction.

\section{Doping dependence of magnon excitations}

Based upon the results about the magnon dispersion described above, many important issues need to be addressed: how does the observed softening correlate with the carrier concentration $(x)$, on-site disorder, and strength of lattice distortion? So far, it seems quite clear that the zone boundary softening occurs in these relatively low- $T_{\mathrm{C}}$ or narrow-band materials, though it is not quite clear for the high- $T_{\mathrm{C}}$ compounds. If indeed the softening as well as the unusual magnon damping mainly occurs in these low- $T_{\mathrm{C}}$ manganites, which have large Jahn-Teller effects, it may be associated with strong spin-lattice/orbital couplings.

In order to further gain insight into the issue of zone boundary softening, Ye et al [21] have recently systematically analysed existing magnon data and taken additional data in the 


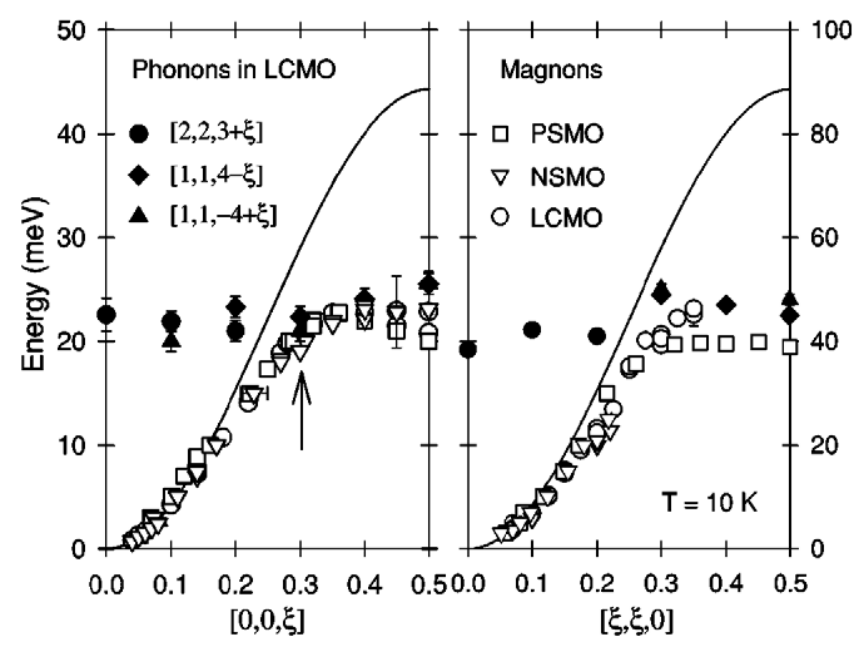

Figure 8. Magnon dispersions (open symbols) of $\mathrm{Nd}_{0.7} \mathrm{Sr}_{0.37} \mathrm{MnO}_{3}, \mathrm{La}_{0.7} \mathrm{Ca}_{0.3} \mathrm{MnO}_{3}$, and $\operatorname{Pr}_{0.63} \mathrm{Sr}_{0.37} \mathrm{MnO}_{3}$ at $T=10 \mathrm{~K}$ along both [0, 0, 1] and [1, 1, 0] directions [27]. Solid symbols show the dispersion of selected LO-phonon modes collected along the reciprocal-lattice directions as specified in the legend.

Table 1. A summary of the fit results of magnon dispersion data to the Heisenberg Hamiltonian with nearest-neighbour $\left(J_{1}\right)$ and fourth-nearest-neighbour $\left(J_{4}\right)$ exchange coupling. The Curie temperature $\left(T_{\mathrm{C}}\right)$, A-site disorder $\left(\sigma^{2}\right)$, spin-wave stiffness $(D), J_{1}, J_{4}$, and their ratio $\left(J_{4} / J_{1}\right)$ are listed. References from which the data are taken are also shown in the column for $D$.

\begin{tabular}{|c|c|c|c|c|c|c|}
\hline Samples & $T_{\mathrm{C}}(\mathrm{K})$ & $\sigma^{2}\left(\times 10^{-3}\right)$ & $D$ [ref.] $\left(\mathrm{meV} \AA^{2}\right)$ & $2 S J_{1}(\mathrm{meV})$ & $2 S J_{4}(\mathrm{meV})$ & $J_{4} / J_{1}(\%)$ \\
\hline \multirow{2}{*}{$\mathrm{La}_{0.7} \mathrm{Sr}_{0.3} \mathrm{MnO}_{3}$} & 378 & 1.8556 & $188[18]$ & & & \\
\hline & & & $176[19]$ & 7.63 & 1.66 & $22 \pm 1.4$ \\
\hline $\mathrm{La}_{0.7} \mathrm{~Pb}_{0.3} \mathrm{MnO}_{3}$ & 355 & 3.7708 & 134 [17] & 8.79 & - & - \\
\hline $\mathrm{La}_{0.7} \mathrm{Ba}_{0.3} \mathrm{MnO}_{3}$ & 350 & 1.3548 & $152[32]$ & 7.30 & 1.42 & $20 \pm 3.2$ \\
\hline $\mathrm{La}_{0.68} \mathrm{Ba}_{0.32} \mathrm{MnO}_{3}$ & 336 & 1.4038 & $-[31]$ & 7.03 & 1.59 & $23 \pm 2.1$ \\
\hline $\mathrm{La}_{0.8} \mathrm{Sr}_{0.2} \mathrm{MnO}_{3}$ & 312 & 1.4138 & 167 [19] & - & - & - \\
\hline $\operatorname{Pr}_{0.63} \mathrm{Sr}_{0.37} \mathrm{MnO}_{3}$ & 301 & 4.0002 & $165[24]$ & 5.16 & 2.08 & $40 \pm 4.4$ \\
\hline $\mathrm{La}_{0.67} \mathrm{Ca}_{0.33} \mathrm{MnO}_{3}$ & 250 & 0.2865 & $170[26]$ & & & \\
\hline $\mathrm{La}_{0.7} \mathrm{Ca}_{0.3} \mathrm{MnO}_{3}$ & 238 & 0.2722 & 165 [27] & 6.63 & 0.76 & $11 \pm 1.4$ \\
\hline $\mathrm{Nd}_{0.7} \mathrm{Sr}_{0.3} \mathrm{MnO}_{3}$ & 198 & 4.5379 & $165[25]$ & - & - & - \\
\hline $\mathrm{La}_{0.75} \mathrm{Ca}_{0.25} \mathrm{MnO}_{3}$ & 191 & 0.2430 & $152[21]$ & 7.90 & 0.36 & $5 \pm 0.5$ \\
\hline $\mathrm{Sm}_{0.55} \mathrm{Sr}_{0.45} \mathrm{MnO}_{3}$ & 135 & 7.8418 & $140[28]$ & 2.91 & 1.74 & $60 \pm 8.9$ \\
\hline $\operatorname{Pr}_{0.7} \mathrm{Ca}_{0.3} \mathrm{MnO}_{3}$ & $\mathrm{a}$ & $2.1 \times 10^{-4}$ & $145[21]$ & 5.98 & 0.61 & $10 \pm 2.6$ \\
\hline $\operatorname{Pr}_{0.55}\left(\mathrm{Ca}_{0.85} \mathrm{Sr}_{0.15}\right)_{0.45} \mathrm{MnO}_{3}$ & $\mathrm{a}$ & 2.0973 & $152[21]$ & 3.24 & 1.79 & $55 \pm 8.1$ \\
\hline
\end{tabular}

a The ground state of these materials is the AF insulating state but can be transformed into the FM state by field cooling.

FM metallic state of $\mathrm{R}_{1-x} \mathrm{~A}_{x} \mathrm{MnO}_{3}$ at judicially selected doping levels. In additional to the single crystals of $\mathrm{La}_{0.75} \mathrm{Ca}_{0.25} \mathrm{MnO}_{3}, \mathrm{La}_{0.7} \mathrm{Ca}_{0.3} \mathrm{MnO}_{3}, \mathrm{Nd}_{0.7} \mathrm{Sr}_{0.3} \mathrm{MnO}_{3}, \mathrm{Pr}_{0.63} \mathrm{Sr}_{0.37} \mathrm{MnO}_{3}$, $\mathrm{La}_{0.7} \mathrm{Sr}_{0.3} \mathrm{MnO}_{3}, \mathrm{Sm}_{0.55} \mathrm{Sr}_{0.45} \mathrm{MnO}_{3}, \mathrm{La}_{0.7} \mathrm{Ba}_{0.3} \mathrm{MnO}_{3}$, and $\mathrm{La}_{0.68} \mathrm{Ba}_{0.32} \mathrm{MnO}_{3}$, which have the FM metallic phase as the ground state, $\operatorname{Pr}_{0.7} \mathrm{Ca}_{0.3} \mathrm{MnO}_{3}$ and $\operatorname{Pr}_{0.55}\left(\mathrm{Ca}_{0.85} \mathrm{Sr}_{0.15}\right)_{0.45} \mathrm{MnO}_{3}$ have also been used for the study. The latter two samples exhibit AF/canted AF insulating ground state but can be tuned into the FM metallic state by applying an external magnetic field (see the phase diagram $[21,33]$ of these two compounds in figure 9), thus the magnon behaviour in the field-induced FM metallic state can also be studied. Though application of magnetic 


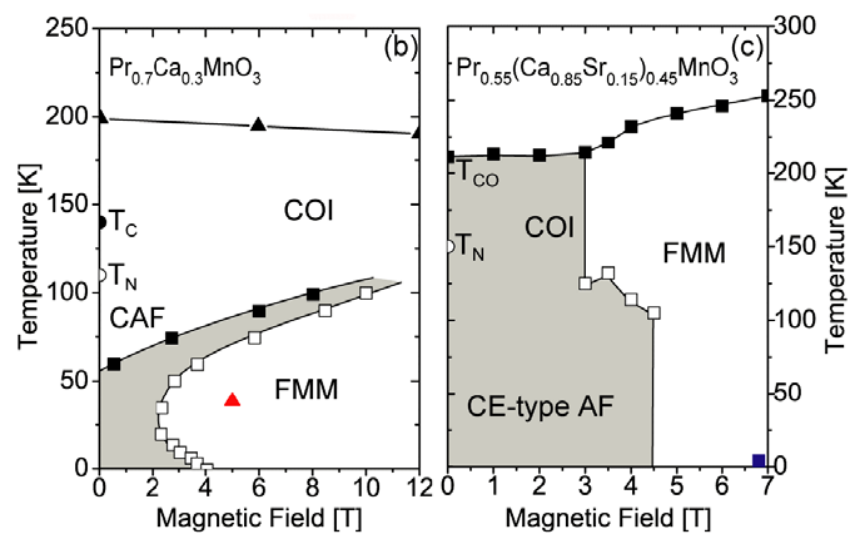

Figure 9. Phase diagrams of $\operatorname{Pr}_{0.7} \mathrm{Ca}_{0.3} \mathrm{MnO}_{3}$ [33] and $\operatorname{Pr}_{0.55}\left(\mathrm{Ca}_{0.85} \mathrm{Sr}_{0.15}\right)_{0.45} \mathrm{MnO}_{3}$ [21] in the $T-H$ plane based on transport measurement. The neutron scattering measurements on magnon excitations were taken at the position marked by the (red) upper triangle and the (blue) square symbols.

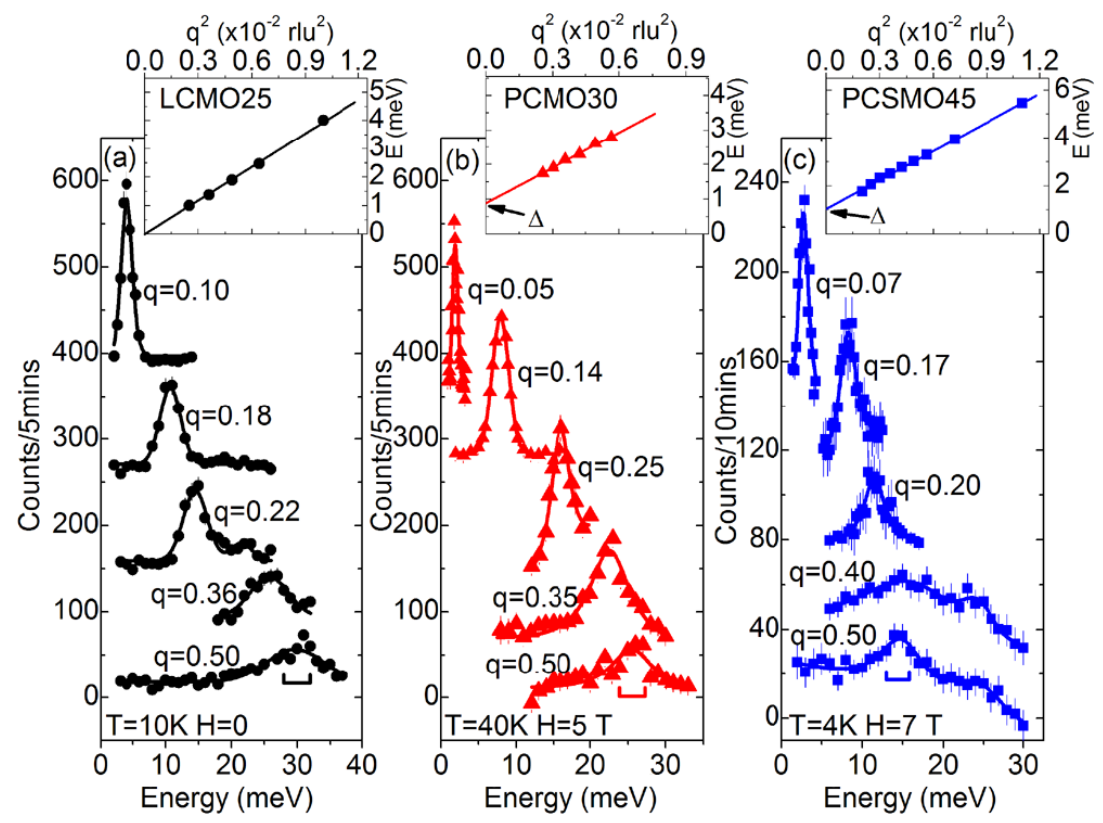

Figure 10. $q$ dependence magnon excitation spectra in (a) $\mathrm{La}_{0.75} \mathrm{Ca}_{0.25} \mathrm{MnO}_{3}$, (b) $\mathrm{Pr}_{0.7} \mathrm{Ca}_{0.3} \mathrm{MnO}_{3}$ under $5 \mathrm{~T}$ magnetic field, and (c) $\operatorname{Pr}_{0.55}\left(\mathrm{Ca}_{0.85} \mathrm{Sr}_{0.15}\right)_{0.45} \mathrm{MnO}_{3}$ under $7 \mathrm{~T}$ magnetic field. The spectra at different $q$ values are incrementally shifted for clarity. The instrumental resolutions are shown in the horizontal bars and the shoulders around $15 \mathrm{meV}$ in (c) are the phonon scattering. $E$ versus $q^{2}$ is plotted in the insets to determine $D$.

field adds a field-induced Zeeman gap [34], it is remarkable that all three samples exhibit very similar low- $q$ behaviour disregarding the difference in achieving the FM metallic states either by temperature or by magnetic field. As shown in the insets of figure 10 [21], the slopes of the magnon energy $(E)$ versus $q^{2}$ lines yield $D$ values of $150 \pm 3,145 \pm 8$, and $152 \pm 3 \mathrm{meV} \AA^{2}$ for $\mathrm{La}_{0.75} \mathrm{Ca}_{0.25} \mathrm{MnO}_{3}, \mathrm{Pr}_{0.7} \mathrm{Ca}_{0.3} \mathrm{MnO}_{3}$, and $\mathrm{Pr}_{0.55}\left(\mathrm{Ca}_{0.85} \mathrm{Sr}_{0.15}\right)_{0.45} \mathrm{MnO}_{3}$, respectively. This 


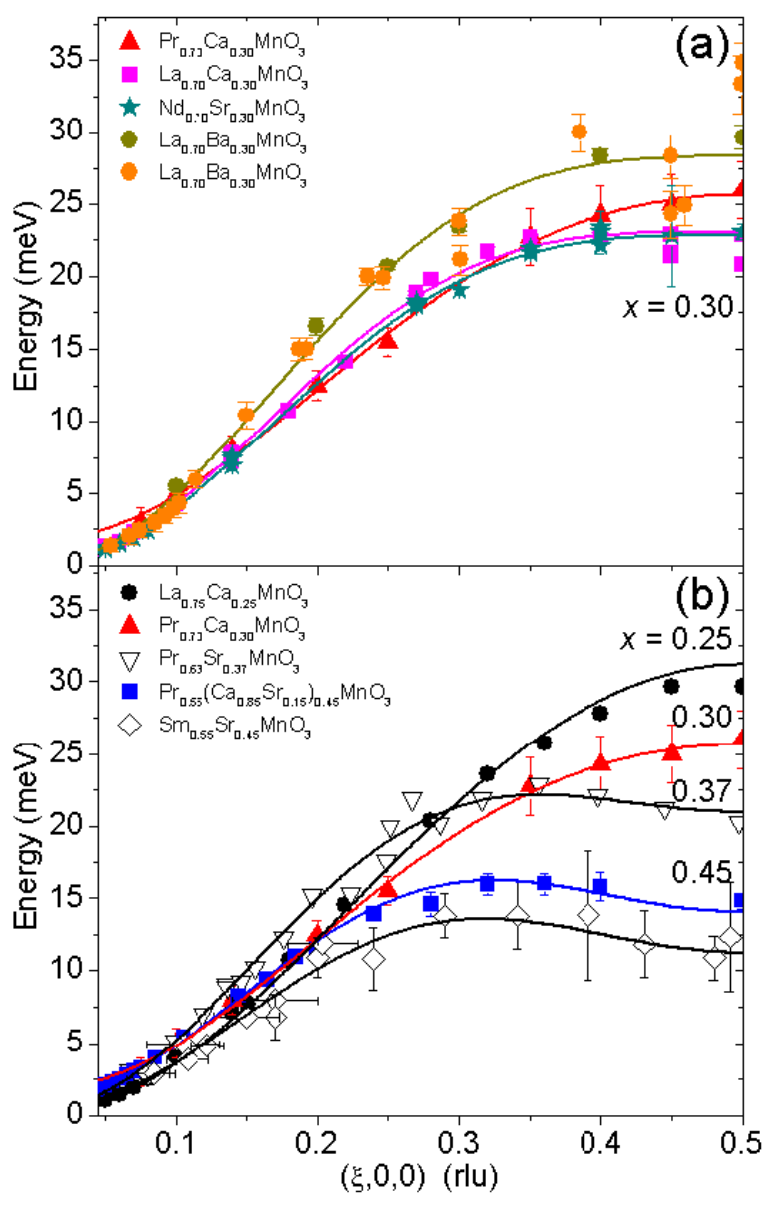

Figure 11. Summary of magnon dispersion curves along the $[1,0,0]$ direction for (a) various $\mathrm{R}_{0.7} \mathrm{~A}_{0.3} \mathrm{MnO}_{3}$ manganites and (b) a series of $\mathrm{R}_{1-x} \mathrm{~A}_{x} \mathrm{MnO}_{3}$ as a function of $x$. The solids are the least-square fits using the Heisenberg model with $J_{1}$ and $J_{4}$. Data are obtained from [19, 21, 24, 25, 27, 28, 32].

clearly indicates that the spin-wave stiffness is independent of how the FM metallic phase is realized or the carrier concentration.

To determine the evolution of magnon excitations in $\mathrm{R}_{1-x} \mathrm{~A}_{x} \mathrm{MnO}_{3}$ as a function of doping, figure 11(a) summarizes the magnon dispersions along the [1, 0, 0] direction for a series of $\mathrm{R}_{1-x} \mathrm{~A}_{x} \mathrm{MnO}_{3}$ with $x \approx 0.3[19,21,25,27,31]$ while figure 11(b) presents the dispersions along the same direction but for different doping concentrations [21, 24, 28]. The solid curves in the figure are phenomenological fits to the data using the Heisenberg Hamiltonian equations (3) and (4) with nearest-neighbour $\left(J_{1}\right)$ and fourth-nearest-neighbour $\left(J_{4}\right)$ exchange coupling. In the low- $q$ limit, $E(q)=\Delta+8 \pi^{2} S\left(J_{1}+4 J_{4}\right) q^{2}$, instead of using equation (5), which takes only the nearest-neighbour coupling. It is found $[21,24,28]$ that the contributions from the second-nearest-neighbour $\left(J_{2}\right)$ and third-nearest-neighbour $\left(J_{2}\right)$ exchange coupling are negligible. While the magnons show similar dispersions for $\mathrm{R}_{1-x} \mathrm{~A}_{x} \mathrm{MnO}_{3}$ with $x=0.3$ (figure 11(a)), the doping dependence of the zone-boundary magnon softening (figure 11(b)) indicates that the higher the doping level, the larger the zone boundary softening.

We should emphasize that besides the doping, the effect of A-site disorder (or chemical disorder) arising from the mismatch between rare- and alkaline-earth-metal ions might induce anomalous spin dynamical behaviour [35-38]. The A-site disorder is characterized by the standard deviation of the ionic radii: $\sigma^{2}=\sum_{i}\left(x_{i} r_{i}^{2}-\bar{r}^{2}\right)$, where $x_{i}$ are the fractional 


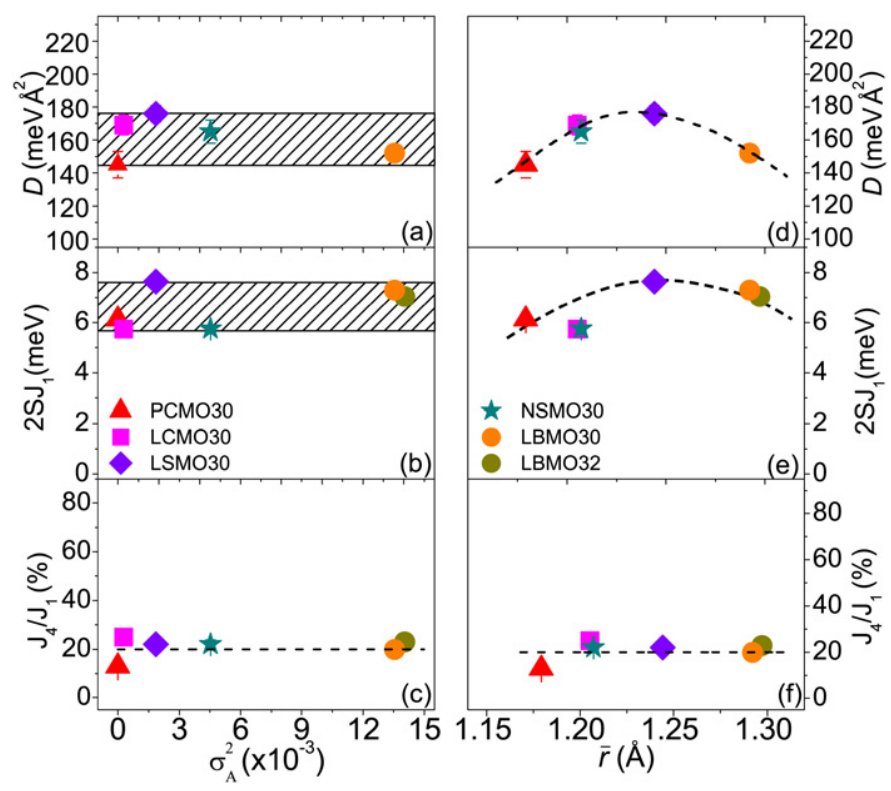

Figure 12. Disorder dependence of (a) the spin-wave stiffness $D$ measured from low$q$ magnon excitations, (b) the nearest-neighbour exchange coupling $2 S J_{1}$ and (c) the ratio $J_{4} / J_{1}$; the average ionic radius dependence of (d) $D$, (e) $2 S J_{1}$, and (f) $J_{4} / J_{1}$. Dashed lines are guides to the eye (from [21]).

occupancies of A-site species and $r_{i}$ and $\bar{r}=\sum_{i} x_{i} r_{i}$ are the individual and averaged ionic radii, respectively [39]. Figure 12 summarizes the $\sigma^{2}$ and $\bar{r}$ dependence of the spin-wave stiffness $D, J_{1}$, and the ratio of $J_{4} / J_{1}$, which accounts for the zone boundary softening [21]. Surprisingly, varying disorder seems to have no systematic effect on $D$ or $J_{1}$. With increasing disorder, the spin-wave stiffness falls within a bandwidth of $D=160 \pm 15 \mathrm{meV} \AA^{2}$ and the nearest-neighbour exchange coupling falls within a bandwidth of $2 S J_{1}=7 \mathrm{meV}$ (figures 12 (a) and (b)). Furthermore, the ratio of $J_{4} / J_{1}$ shows no dependence on the on-site disorder (figure 12(c)), in contrast with the recent theoretical prediction [35] suggesting a significant zone boundary softening with increasing disorder.

On the other hand, both $D$ and $2 S J_{1}$ show a different dependence on the average ionic radius $\bar{r}$ at A-sites. As shown in figures $12(\mathrm{~d})$ and (e), both $D$ and $2 S J_{1}$ do show a parabolic curve but with a small bandwidth. This is certainly a puzzle for the understanding of the spin dynamics in FM manganites. Changing the ionic size at A site will modify the length and angle of $\mathrm{Mn}-\mathrm{O}-\mathrm{Mn}$ bonds, thus leading to changes in effective transfer integral between $\mathrm{Mn}$ ions or the bandwidth of the electrons [40]. Despite the large change in $T_{\mathrm{C}}$ by varying the average ionic radius, the kinetic energy $(D)$ or the bandwidth of the electrons seems to change only slightly based on these results, in consistence with earlier studies [25, 27, 41]. Moreover, $J_{4} / J_{1}$ shows no dependence on $\bar{r}$ (figure 12(f)), thus indicating that the zone-boundary magnon softening is independent of $T_{\mathrm{C}}$ as a general feature of the $\mathrm{FM} \mathrm{R}_{1-x} \mathrm{~A}_{x} \mathrm{MnO}_{3}$ manganites.

To gain more insight into the doping dependence of spin dynamics, figure 13 plots the measured values of $D, 2 S J_{1}$, and $J_{4} / J_{1}$ as a function of doping [21], respectively. For $\mathrm{La}_{0.8} \mathrm{Sr}_{0.2} \mathrm{MnO}_{2}$, the value of $D=166.8 \pm 1.51 \mathrm{meV} \AA^{2}$ is used here from a more accurate measurement [19]. In contrast with earlier results [20] (see figure 4), the spin-wave stiffness $D$ keeps a value around $160 \pm 15 \mathrm{meV} \AA^{2}$ and is essentially unchanged for the doping range of $0.2 \leqslant x \leqslant 0.45$ (see figure $13(\mathrm{a})$ ) while $T_{\mathrm{C}}$ varies in a wide range from $135 \mathrm{~K}$ for $\mathrm{Sm}_{0.55} \mathrm{Sr}_{0.45} \mathrm{MnO}_{3}$ [28] to $378 \mathrm{~K}$ for $\mathrm{La}_{0.7} \mathrm{Sr}_{0.3} \mathrm{MnO}_{3}$ [19]. This is also in contrast with the theoretical predication based upon the $1 / S$ spin-wave expansion for DE ferromagnets by Golosov et al [12]. 


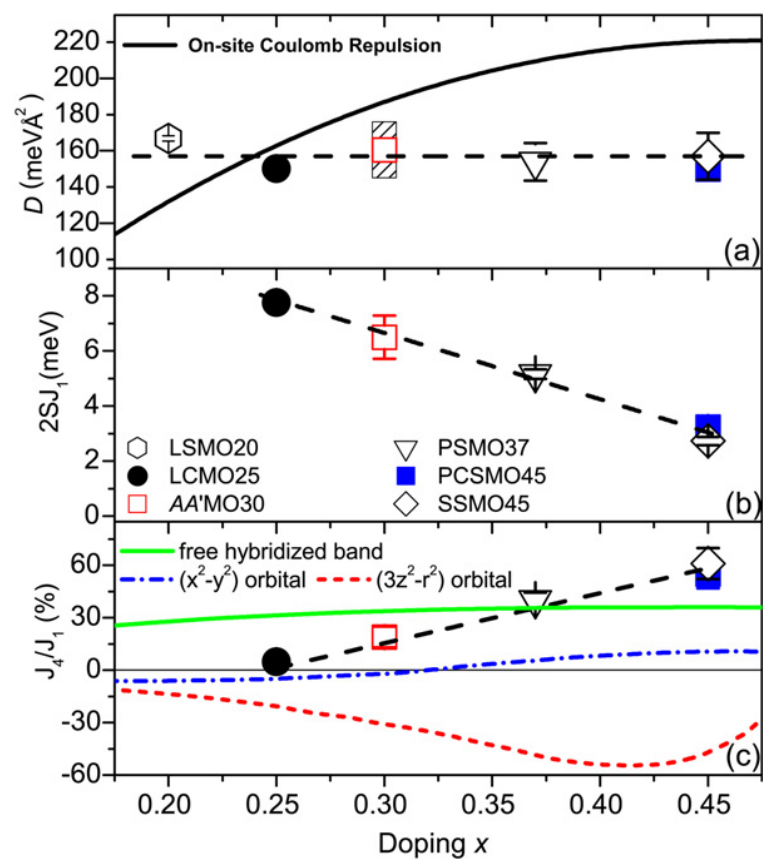

Figure 13. Doping $(x)$ dependence of (a) $D$, (b) $2 S J_{1}$, and (c) $J_{4} / J_{1}$. Dashed lines are guides to the eye. The solid curve in (a) is the prediction of [11]. The (green) solid line in (c) is the calculation result from [42] while (blue) dash-dotted and (red) dashed lines in (c) are these from [28]. The figure is taken from [21].

Conversely, the nearest-neighbour exchange coupling $J_{1}$ and the ratio $J_{4} / J_{1}$ do show a linear-type relation with the doping concentration. $2 S J_{1}$ decreases while $J_{4} / J_{1}$ increases, approximately linearly, with increasing $x$ (figures 13(b) and (c)). This clearly shows that the zone-boundary magnon softening (denoted by the ratio $J_{4} / J_{1}$ ) is enhanced linearly with increasing doping. However, as shown in figure 13(c), the $x$ dependence of the ratio $J_{4} / J_{1}$ cannot be accounted for by the recent proposal [28] based upon the mechanism of the $d_{3 z^{2}-r^{2}-}$ or $\mathrm{d}_{x^{2}-y^{2}}$-type orbital fluctuations or the free hybridized band model suggested by Solovyev et al [42] (see section 9 for more discussion). The change of the ratio versus doping from the free hybridized band model is too small to account for the experimental results, while the orbital fluctuation model gives a non-monotonic doping dependence of the ratio. The simple linear relation of the ratio $J_{4} / J_{1}$ to doping deserves further careful investigation.

\section{Anomalous magnon damping}

Right after the measurements on the magnon behaviour in FM manganites with inelastic neutron scattering, it was discovered [24, 26, 27] that the magnon excitation spectra have unusual large linewidths, especially near the zone boundary. As shown in figure 7, the magnon excitation peaks show a large increase of linewidth and damping when the reduced wavevector $\xi>0.3$ up to the zone boundary at $\xi=0.5$. Similar behaviour has also been observed in $\mathrm{Nd}_{0.6} \mathrm{Sr}_{0.4} \mathrm{MnO}_{3}$ [43]. Figure 14 plots the intrinsic linewidths (FWHM) of the magnon peaks along the $[0,0,1]$ direction for three manganites at $10 \mathrm{~K}: \operatorname{Pr}_{0.63} \operatorname{Sr}_{0.37} \mathrm{MnO}_{3}\left(T_{\mathrm{C}}=301 \mathrm{~K}\right)$, $\mathrm{La}_{0.7} \mathrm{Ca}_{0.3} \mathrm{MnO}_{3}\left(T_{\mathrm{C}}=238 \mathrm{~K}\right)$, and $\mathrm{Nd}_{0.7} \mathrm{Sr}_{0.3} \mathrm{MnO}_{3}\left(T_{\mathrm{C}}=198 \mathrm{~K}\right)$ [27]. Near the zone centre, the linewidth reaches almost the instrumental limit while a drastic increase at a wavevector larger than $\xi \sim 0.3$. More interestingly, the linewidths from all three samples show similar behaviour, indicating a possible common mechanism for the effect on the magnon lifetime near the zone boundary regardless of the size of the difference in the Curie temperature. Meanwhile, 


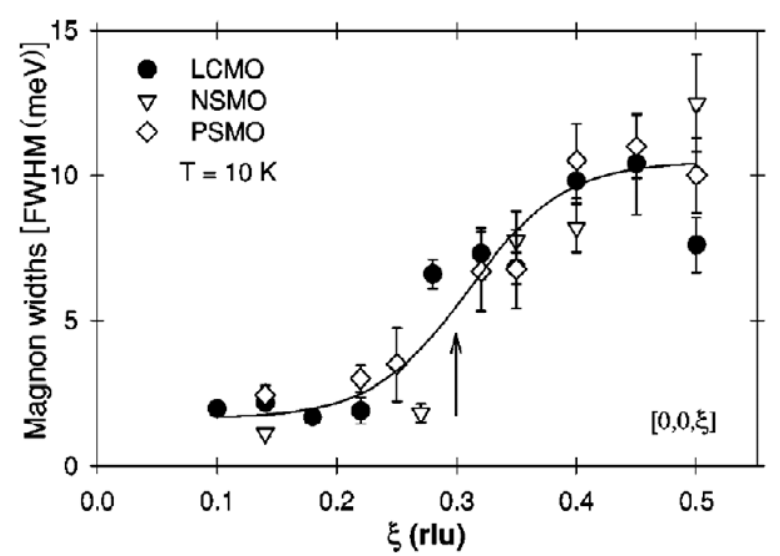

Figure 14. Linewidth of magnon excitation spectra along the $[1,0,0]$ direction for $\mathrm{Nd}_{0.7} \mathrm{Sr}_{0.37} \mathrm{MnO}_{3}, \mathrm{La}_{0.7} \mathrm{Ca}_{0.3} \mathrm{MnO}_{3}$, and $\mathrm{Pr}_{0.63} \mathrm{Sr}_{0.37} \mathrm{MnO}_{3}$ at $T=10 \mathrm{~K}$. a significant magnon linewidth broadening is seen after $\xi \geqslant 0.3$ (marked by arrows), when an optical phonon merges with the magnon. The figure is taken from [27].

it has been found that the strong magnon damping near the zone boundary has a dependence on $T_{\mathrm{C}}$, although a systematic study is still needed. For example, although still relatively well defined throughout the Brillouin zone in the $[1,0,0]$ direction for both compounds, the magnon excitations are much more severely damped along the $[1,1,0]$ direction for $\mathrm{Nd}_{0.7} \mathrm{Sr}_{0.3} \mathrm{MnO}_{3}$ than for $\mathrm{La}_{0.7} \mathrm{Ca}_{0.3} \mathrm{MnO}_{3}$ [27]. Some preliminary measurements on several manganites [44] suggest that lower- $T_{\mathrm{C}}$ manganites have larger zone boundary magnon damping.

Generally, the predominant effect on magnon linewidth is magnon-magnon scattering such that in the long-wavelength regime the linewidth obeys a certain scaling law as $\Gamma_{\text {mag }} \propto$ $q^{4} \ln ^{2}\left(k_{\mathrm{B}} T / \hbar \omega_{q}\right)$ for $\hbar \omega_{q} \ll k_{\mathrm{B}} T$, and $\Gamma \propto q^{3}$ for $\hbar \omega_{q} \gg k_{\mathrm{B}} T$ [45, 23]. However, the observed sudden increase in magnon linewidth and heavy damping near the zone boundary certainly deviates from the simple scaling law [23, 27]. Such magnon behaviour should be attributed to a mechanism other than Heisenberg-type interactions.

In the low-temperature FM metallic phase, a possible mechanism accounting for the magnon broadening is due to the Stoner continuum, where magnons decay and cause electronhole excitations. However, the FM ground state of manganite, especially if the system is indeed in the half-metallic phase, would have a complete separation of the majority and minority bands due to the large Hund's-rule coupling $J_{\mathrm{H}}$. As a consequence, the Stoner continuum is expected to lie at an energy scale $\left(2 J_{\mathrm{H}}\right)$ much higher than that of the magnon excitations. It seems unlikely that the magnon broadening and damping are caused by the Stoner continuum excitations [10].

As reported by Dai et al [27], the unusual magnon broadening/damping as well as softening may indicate a possible magnon-phonon coupling [25, 46, 47]. As shown in figure 15(a) for the measured results from $\mathrm{La}_{0.7} \mathrm{Ca}_{0.3} \mathrm{MnO}_{3}$, the two particular optical phonon modes $\left(\Omega_{1}\right.$ and $\Omega_{2}$, respectively) which are characterized as two vibration modes associated with the $\mathrm{MnO}_{6}$ octahedron [48, 49] merge with the magnons in both [1, 0, 0] and [1, 1, 0] directions (see also figure 8). Evidently, the in-plane magnons exhibit softening and broadening when they merge with the phonons around the momentum transfer $\xi=0.3$ for both directions. To clearly show the correlation between magnons and phonons in the excitation spectrum, we plot in figure 14(b) the magnon linewidth as a function of magnon energy. In the $[1,0,0]$ direction, the magnon softens and simultaneously increases its linewidth abruptly from $\sim 4$ to $\sim 12 \mathrm{meV}$ 

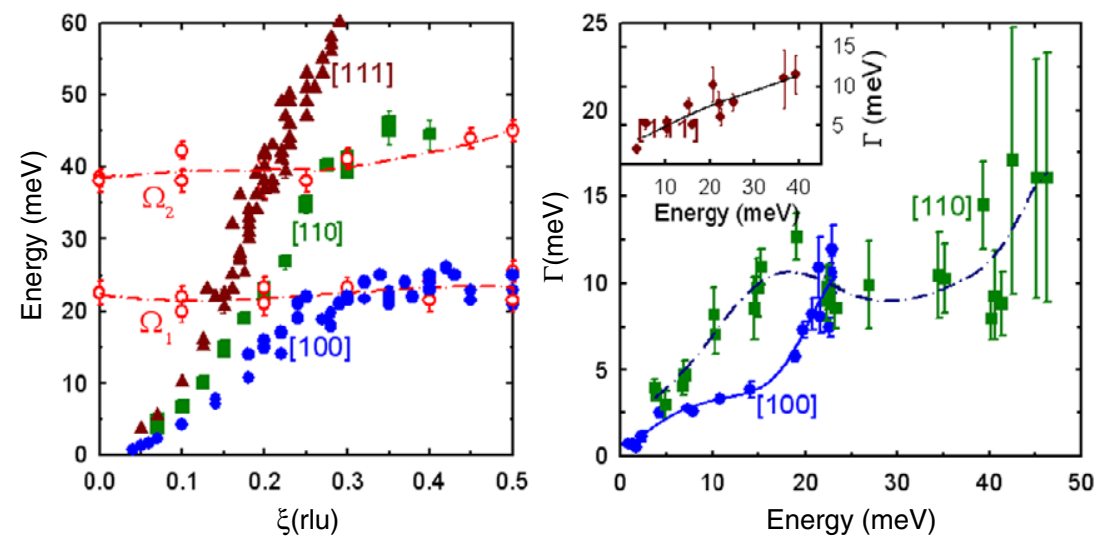

Figure 15. (A) Dispersion of magnons (solid symbols) along the $[1,0,0],[1,1,0]$, and $[1,1,1]$ directions and two related optical phonon modes (open symbols) along the $[1,0,0]\left(\Omega_{1}\right)$ and $[1,1$, 0] $\left(\Omega_{2}\right)$ directions of $\mathrm{La}_{0.7} \mathrm{Ca}_{0.3} \mathrm{MnO}_{3}$ at $T=10 \mathrm{~K}$; (B) the linewidth of magnon excitation spectra as a function of magnon energy (bottom) of $\mathrm{La}_{0.7} \mathrm{Ca}_{0.3} \mathrm{MnO}_{3}$ at $T=10 \mathrm{~K}$. The inset shows the magnon linewidth as a function of energy in the $[1,1,1]$ direction. The lines are guides to the eye.

at $\xi=0.3$, where the magnon merges with the $\Omega_{1}$ phonon at energy around $20 \mathrm{meV}$. In the [1, $1,0]$ direction, the linewidth of the magnon exhibits a peak/shoulder around $20 \mathrm{meV}$ where the magnon disperses across with the $\Omega_{1}$ phonon. Furthermore, when merging with the $\Omega_{2}$ phonon around $\xi=0.3$ and the energy of $45-50 \mathrm{meV}$, the magnon damps drastically and increases its linewidth abruptly. The large error bar in the linewidth near the zone boundary in the [1, $1,0]$ direction is indeed because of the low peak intensity in the excitation spectra due to the significant magnon damping when the magnon probably entangles with the $\Omega_{2}$ phonon branch. Furthermore, such drastic magnon damping close to the zone boundary is much more enhanced for lower- $T_{\mathrm{C}}$ samples and in the $[1,1,0]$ than $[1,0,0]$ direction, indicating an anisotropy of magnon damping even in the $\mathrm{MnO}_{2}$ plane [23, 27, 44]. Actually, in the case of $\mathrm{Nd}_{0.7} \mathrm{Sr}_{0.3} \mathrm{MnO}_{3}$, which has lower $T_{\mathrm{C}}$ than $\mathrm{La}_{0.7} \mathrm{Ca}_{0.3} \mathrm{MnO}_{3}$, the zone-boundary magnons are overdamped to be experimentally measured [27].

It is worthy to mention that the $\Omega_{2}$ phonon merging with the $[1,1,0]$ magnon branch is a JT-active mode associated with the oxygen vibration in the $\mathrm{MnO}_{6}$ octahedron while the $\Omega_{1}$ phonon is an external mode associated with the La vibration against the $\mathrm{MnO}_{6}$ octahedron. If spin-phonon interaction is responsible for the broadening/damping of the magnon, the difference in the character of these two phonon modes should directly relate to the observed difference in damping of the in-plane magnons between the $[1,0,0]$ and $[1,1,0]$ directions. In contrast, in the $[1,1,1]$ direction, the magnon shows no obvious and unusual behaviour (see the inset of figure 15(b)). These results indicate that the anomalous broadening and damping behaviour of the in-plane but not the out-of-the-plane magnons occurs when the magnons and optical phonons merge in the energy-momentum space.

Nevertheless, more quantitative measurements on the intrinsic linewidth of magnon excitations are clearly needed. Even for the magnon behaviour in the [1,0,0] direction, which has been most widely studied, the measured results on linewidths near the zone boundary are still controversial. The results measured from $\mathrm{Sm}_{0.55} \mathrm{Sr}_{0.45} \mathrm{MnO}_{3}$ [28] show the magnon linewidth along the $[1,0,0]$ direction is within $0.7 \mathrm{meV}$ (instrumental energy resolution limited), more than one order smaller than the results from other groups [23, 24, 27]. It was claimed that there is neither anomalous broadening of the magnon spectra nor loss of the 


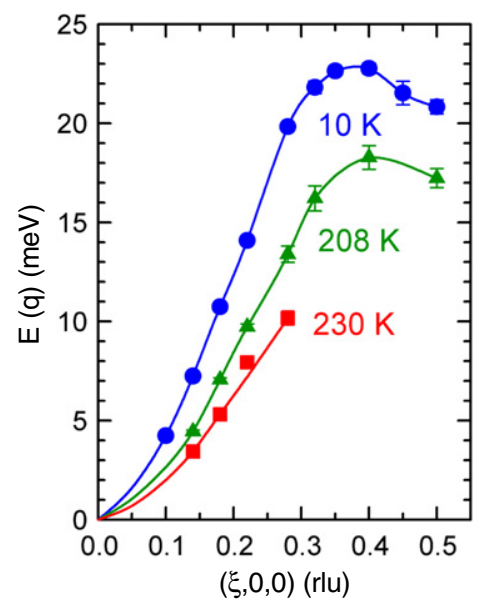

Figure 16. $T$ dependence of magnon dispersion along the $[1,0,0]$ direction of $\mathrm{La}_{0.7} \mathrm{Ca}_{0.3} \mathrm{MnO}_{3}$. The lines are guides to the eye.

scattering intensities in $q_{\mathrm{s}}$ where the dispersion tends to show softening, thus excluding level crossing with phonons or phase separation. In order to identify the nature of the finite lifetime of magnons, especially that close to the zone boundary, a polarized neutron scattering experiment is ideal, which allows a complete separation of the contribution from other excitations like phonons [50].

\section{Temperature dependence and incoherent spin dynamics near $T_{\mathrm{C}}$}

The $T$ dependence of spin dynamics in the FM metallic manganites is reflected by the evolution of the magnon dispersion including the spin-wave stiffness as a function of temperature as well as some unusual incoherent spin dynamics as $T \rightarrow T_{\mathrm{C}}$. The earlier measurement on $\operatorname{Pr}_{0.63} \mathrm{Sr}_{0.37} \mathrm{MnO}_{3}$ (see figure 5) [24] shows that the magnon dispersion relation uniformly softens with increasing temperature. Figure 16 shows the measured $T$ dependence of the magnon dispersion along the $[1,0,0]$ direction from $\mathrm{La}_{0.7} \mathrm{Ca}_{0.3} \mathrm{MnO}_{3}$, further confirming the gradual softening with increasing temperature. However, the temperature has non-uniform effects on the magnon lifetime $[23,24]$. While there is no obvious effect near the zone centre, magnons near the zone boundary show substantial increase in linewidth and decrease in intensity with increasing $T$. For example, as shown in figure 17, the zone boundary linewidths in $\operatorname{Pr}_{0.63} \mathrm{Sr}_{0.37} \mathrm{MnO}_{3}$ along the $[1,0,0]$ direction are nearly doubled from their value of $8.4 \pm 0.5 \mathrm{meV}$ at $T=10 \mathrm{~K}$ to $13.2 \pm 1.9 \mathrm{meV}$ at $T=265 \mathrm{~K}$. For $\mathrm{La}_{0.7} \mathrm{Ca}_{0.3} \mathrm{MnO}_{3}$, which has a lower $T_{\mathrm{C}}$ compared with $\operatorname{Pr}_{0.63} \mathrm{Sr}_{0.37} \mathrm{MnO}_{3}$, the magnons near the zone boundary are overdamped when $T \rightarrow T_{\mathrm{C}}$ such that no reliable dispersion data can be obtained along the [1, $0,0]$ direction when $\xi \geqslant 0.3$. Such $T$-induced broadening and damping near the zone boundary are even severe in the $[1,1,0]$ direction and for lower $T_{\mathrm{C}}$ manganites [44]. This indicates that there is another effect causing the enhanced magnon damping near the zone boundary rather than the simple magnon-magnon scattering and such effects should be $T_{\mathrm{C}}$ dependent.

Even in the long-wavelength (low- $q$ ) limit, the $T$ dependence of magnons also shows unusual behaviour, which is clearly dependent on $T_{\mathrm{C}}[19,23,25,26,51,52]$. It is found [25, 26] that, for low- $T_{\mathrm{C}}$ samples, the spin-wave stiffness $D(T)$ exhibits a power law behaviour as a function of temperature but does not collapse as $T \rightarrow T_{\mathrm{C}}$, thus challenging the simple theories based on a Heisenberg ferromagnetism and DE model. For a Heisenberg ferromagnet, $D(T)$ is expected to follow mode-mode coupling theory [53] with $D(T)=D(0)\left(1-A T^{5 / 2}\right)$ at low $T / T_{\mathrm{C}}$. As $T \rightarrow T_{\mathrm{C}}, D(T)$ should renormalize to zero at $T_{\mathrm{C}}$ with power law behaviour like 


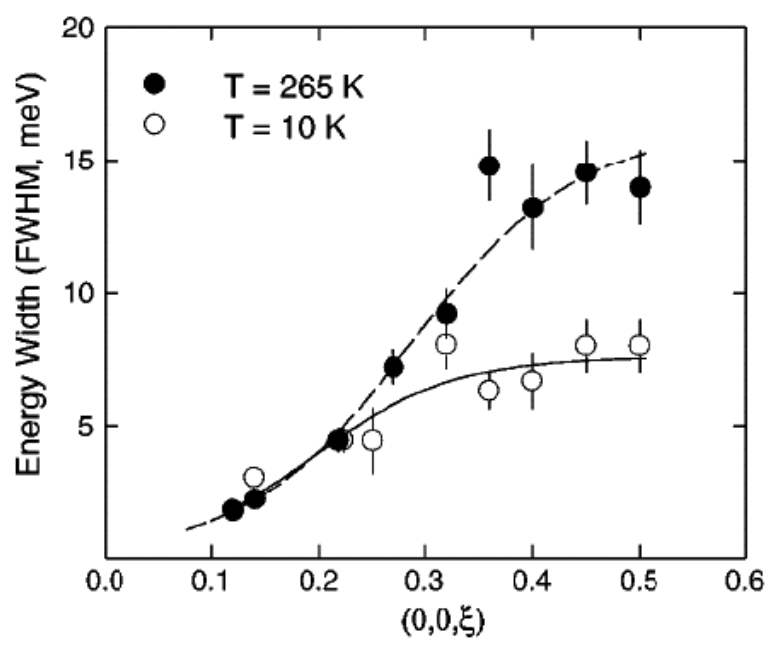

Figure 17. $q$ dependence of magnon linewidths of $\operatorname{Pr}_{0.63} \mathrm{Sr}_{0.37} \mathrm{MnO}_{7}$ along the [0, $0,1]$ direction at $T=10$ and $265 \mathrm{~K}$. The solid and dashed lines are guides to the eye. The figure is taken from [22].

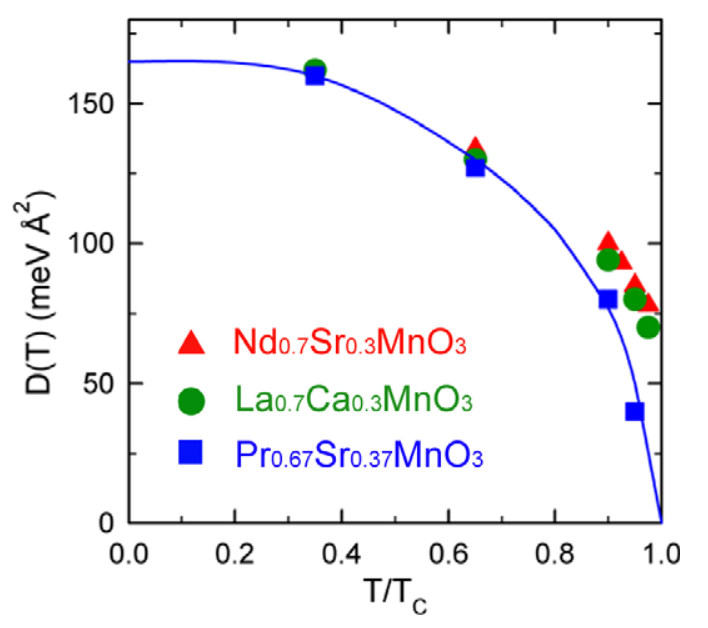

Figure 18. $\left(T / T_{\mathrm{C}}\right)$ dependence of spin-wave stiffness $D\left(T / T_{\mathrm{C}}\right)$ in $\mathrm{Nd}_{0.7} \mathrm{Sr}_{0.37} \mathrm{MnO}_{3} \quad\left(T_{\mathrm{C}}=\right.$ $198 \mathrm{~K}), \mathrm{La}_{0.7} \mathrm{Ca}_{0.3} \mathrm{MnO}_{3}\left(T_{\mathrm{C}}=238 \mathrm{~K}\right)$, and $\mathrm{Pr}_{0.63} \mathrm{Sr}_{0.37} \mathrm{MnO}_{3}\left(T_{\mathrm{C}}=301 \mathrm{~K}\right)$. A discontinuity in $D$ as $T \rightarrow T_{\mathrm{C}}$ is obvious for $\mathrm{Nd}_{0.7} \mathrm{Sr}_{0.37} \mathrm{MnO}_{3}$ and $\mathrm{La}_{0.7} \mathrm{Ca}_{0.3} \mathrm{MnO}_{3}$. The solid line is a guide to the eye.

$\left[\left(T-T_{\mathrm{C}}\right) / T_{\mathrm{C}}\right]^{\nu-\beta}$ with $v-\beta=0.34$ [54]. Figure 18 presents the measured $D(T)$ versus $T / T_{\mathrm{C}}$ for three samples: $\mathrm{Nd}_{0.7} \mathrm{Sr}_{0.3} \mathrm{MnO}_{3}\left(T_{\mathrm{C}}=198 \mathrm{~K}\right), \mathrm{La}_{0.7} \mathrm{Ca}_{0.3} \mathrm{MnO}_{3}\left(T_{\mathrm{C}}=238 \mathrm{~K}\right)$, and $\mathrm{Pr}_{0.63} \mathrm{Sr}_{0.37} \mathrm{MnO}_{3}\left(T_{\mathrm{C}}=301 \mathrm{~K}\right)$. The measured $D(T)$ for $\operatorname{Pr}_{0.63} \mathrm{Sr}_{0.37} \mathrm{MnO}_{3}$ almost follows the theoretical expectation from a Heisenberg ferromagnet [25]. However, for $\mathrm{La}_{0.7} \mathrm{Ca}_{0.3} \mathrm{MnO}_{3}$ and $\mathrm{Nd}_{0.7} \mathrm{Sr}_{0.3} \mathrm{MnO}_{3}$, which have lower $T_{\mathrm{C}}$, it seems that $D(T)$ shows no evidence of the magnon collapse at $T_{\mathrm{C}}$, although the magnetization $M(T)$ of these two compounds does not show unusual behaviour.

To further characterize the spin dynamics of FM manganites when $T \rightarrow T_{\mathrm{C}}$ several groups have studied the spin diffuse scattering near $T_{\mathrm{C}}[19,23,25,26,51,52,55,56]$. An anomalous and field-dependent central diffusive component which develops above $T \sim 0.8 T_{\mathrm{C}}$ for $\mathrm{La}_{0.7} \mathrm{Ca}_{0.3} \mathrm{MnO}_{3}$ [25] and $T \sim 0.9 T_{\mathrm{C}}$ for $\mathrm{Nd}_{0.7} \mathrm{Sr}_{0.3} \mathrm{MnO}_{3}$ [23] and dominates the fluctuation spectrum as $T \rightarrow T_{\mathrm{C}}[25,23]$ has been observed for the low- $T_{\mathrm{C}}$ samples, coinciding with the non-collapse behaviour of $D(T)$. This central component is the result of quasi-elastic spin diffuse scattering. Figure 19 presents the $T$ and field dependence of the central diffusive component as well as the magnon peaks measured from $\mathrm{La}_{0.67} \mathrm{Ca}_{0.33} \mathrm{MnO}_{3}$. The central component decreases while the magnon component increases in intensity with increasing field, 

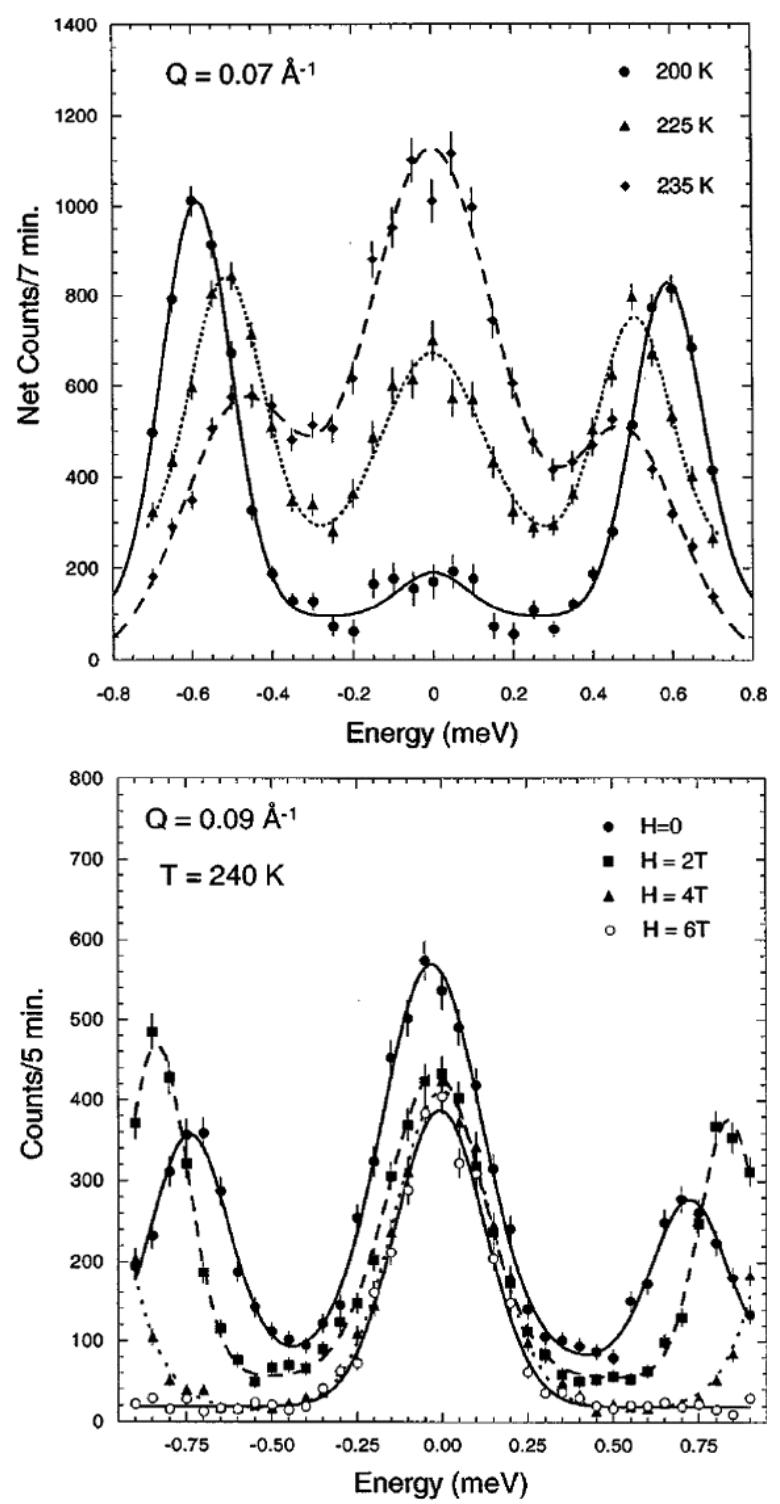

Figure 19. Top, $T$ dependence below $T_{\mathrm{C}}=$ $250 \mathrm{~K}$; bottom, field dependence at $T=$ $240 \mathrm{~K}$ of magnetic inelastic neutron scattering spectra for the $\mathrm{La}_{2 / 3} \mathrm{Ca}_{1 / 3} \mathrm{MnO}_{3}$ polycrystal. The figure is taken from [52]. The central peak is due to quasi-elastic spin diffuse scattering while the two side peaks are due to the magnon excitations. Similar results have been obtained from the $\mathrm{La}_{0.7} \mathrm{Ca}_{0.3} \mathrm{MnO}_{3}$ single crystal [55]. The curves are fits to the data.

thus the strength of the spectrum shifts from the central component into the magnon one as the field is increased. Meanwhile, it is found $[25,26,51,52,56]$ that the temperature at which the central component appears is related to $T_{\mathrm{C}}$. In $\operatorname{Pr}_{0.63} \mathrm{Sr}_{0.37} \mathrm{MnO}_{3}$ the central component emerges only when $T>0.95 T_{\mathrm{C}}$ [25], thus very close to $T_{\mathrm{C}}$.

This anomalous central component has been interpreted [26] as the signature of the formation of spin polarons similar to the ferromagnetic 'droplets' observed in the $\mathrm{La}_{1-x} \mathrm{Ca}_{x} \mathrm{MnO}_{3}$ with lower doping levels [57], thus suggesting a magnetic phase inhomogeneity near $T_{\mathrm{C}}$ [58]. Such a phase inhomogeneity scenario should be invoked for understanding the unusual magnon behaviour and transport properties in manganites including CMR effects. Remarkably, the central diffusive component maximizes its intensity very close to $T_{\mathrm{C}}$, in a manner similar to the evolution of resistivity as well as the lattice 


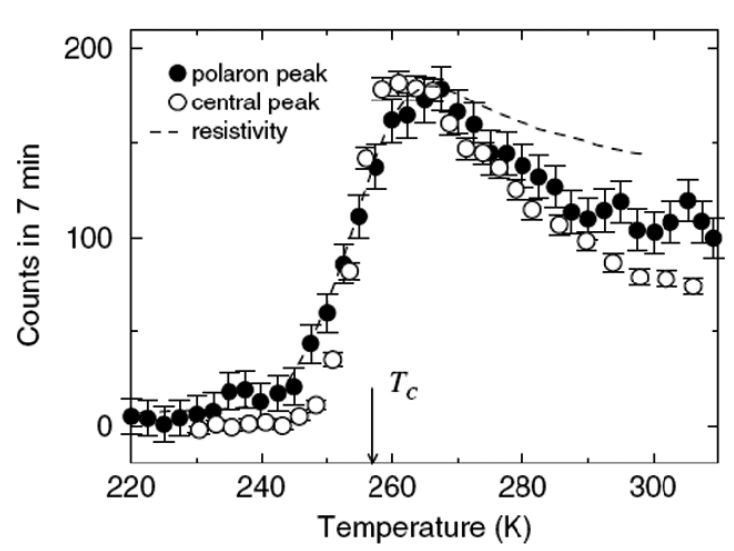

Figure 20. $T$ dependence of the intensity of the central diffusive scattering peak of the $\mathrm{La}_{0.7} \mathrm{Ca}_{0.3} \mathrm{MnO}_{3}$ single crystal, compared with that of the polaron peak at a wavevector of $Q=$ $(3.75,0.25,0)$, and with the sample resistivity. The data have been scaled so that the peak heights match. The similarity of the data suggests a common physical origin. The figure is taken from [55]. Similar results have been obtained in [59].

polarons $[26,55,59]$. Figure 20 shows that the $T$ dependence of the central component at $\boldsymbol{Q}=(1.03,0,0)$ as well as the lattice polaron satellite peak at $Q=(3.75,0.25,0)$ through the FM phase transition is virtually identical to the evolution of resistivity in $\mathrm{La}_{0.7} \mathrm{Ca}_{0.3} \mathrm{MnO}_{3}$, indicating that they all have a common origin which could be related to phase separation. Undoubtedly, such phase inhomogeneities should drastically affect the magnon lifetime, reflected by the unusual damping and the evolution of linewidth of magnons as $T \rightarrow T_{\mathrm{C}}$.

\section{Discussion of possible explanations}

The main issues for the understanding of magnons and associated spin dynamics in FM half-metallic manganites include the unusual $D-T_{\mathrm{C}}$ relation, the anisotropic zone boundary softening and its dependence on doping, and A-site disorder, as well as the anomalous zone boundary broadening/damping. There are quite a few theoretical studies attempting to explain these unusual magnon behaviours. Most of them have focused on the magnon softening and broadening. Although the DE interaction is still the basic ingredient for the understanding of spin dynamics in the FM metallic manganites, at least three major classes of theoretical approaches beyond the canonical DE interaction haven been proposed. The first class is based on the DE interaction and under the ferromagnetic Kondo lattice model, considering the effects of finite Hund's coupling [60], quantum and thermal corrections [12], on-site Coulomb repulsion [11], three-body correlation [61], conducting electron band $\left(\mathrm{e}_{\mathrm{g}}\right)$ filling dependence of the DE and superexchange interactions $[42,62]$, and the non-Stoner continuum in the DE model [63]. The second class emphasizes the effect due to the quantum fluctuations of different $\mathrm{e}_{\mathrm{g}}$-orbitals $[64,65,28]$. The third one goes beyond the electronic origin by taking into account magnon-phonon coupling $[46,47,66]$ and other lattice-related effects such as A-site disorder in the spin excitations [35].

As we already described in section 2, the first attempt using an effective Kondo lattice model $[9,10]$ under the limit of $t / J_{\mathrm{H}} \rightarrow 0$, which is equivalent to the nearest-neighbour FM Heisenberg model, gives a reasonable explanation of the magnon dispersion in $\mathrm{La}_{0.7} \mathrm{~Pb}_{0.3} \mathrm{MnO}_{3}$. This simple model fails to describe the anomalous magnon behaviours in lower- $T_{\mathrm{C}}$ manganites, including the $D-T_{\mathrm{C}}$ relation and zone boundary softening and broadening. However, Shannon 
et al [12] argued that the DE ferromagnet, with the ferromagnetic interaction between core spins mediated by conduction electrons (Kondo-type coupling), is generally not equivalent to the FM Heisenberg model. Specifically, the simple Heisenberg model is valid only when magnons are non-interacting quasiparticles and the spin waves are exact eigenstates of the Heisenberg Hamiltonian. Both quantum and thermal corrections to the magnetic properties of a DE model differ from any effective Heisenberg model because its spin excitations interact only indirectly, through the exchange of charge fluctuations. These new corrections do explain a doping- $(x-)$ dependent zone-boundary magnon softening as compared to that in a Heisenberg ferromagnet. Yet, the corrections also predict a relative magnon hardening in the $[1,1,1]$ direction at $T=0 \mathrm{~K}$, which apparently is inconsistent with experimental observations [27].

Golosov [11] constructed a $1 / S$ spin wave expansion [9] for DE ferromagnets with a sufficiently large Hund's rule coupling in an FM metallic ground state taking into consideration the on-site Coulomb repulsion [67]. He found that magnon-electron scattering, which gives rise to the subleading terms in the $1 / S$ expansion, could provide corrections to the magnon dispersion and damping as well as their momentum dependences, which are mediated by the Fermi surface geometry. In particular, it was found that the magnon linewidth $\Gamma(\vec{q})$ in the longwavelength limit is proportional to $q^{6}$ in a three-dimensional system such as $\mathrm{R}_{1-x} \mathrm{~A}_{x} \mathrm{MnO}_{3}$. This result is in agreement with the calculation by Shannon et al [12] but different from the $q^{4}$ dependence based on magnon-magnon scattering [45]. Furthermore, it was predicted that an anomaly of $\Gamma(\vec{q})$ reflected by either a jump or a logarithmic divergence should occur at $q=k_{\mathrm{F}}$. This seems in agreement with the observation of the magnon linewidth anomaly near $\xi \sim 0.3$ [27]. However, the prediction of a strong doping dependence of $D$ seems inconsistent with the experimental result (see figure 13(a)) [21]. Also, the lack of detailed knowledge of the electronic band structure, especially the lack of reliable experimental data, prevents a quantitative comparison between theoretical and experimental results.

Another possible source to renormalize the self-energy of magnon quasiparticles from the simple DE model is the excitations of 'non-Stoner' continuum states. As we mentioned in section 7, a prevalent view is that in FM half-metallic manganites the Stoner continuum (in the single-spin-flip channel) lies completely above the magnon energies due to larger Hund'srule coupling, thus keeping magnons from decaying into the continuum. However, exact diagonalization studies of the DE model by Kaplan et al indicate that this prevalent picture is incorrect. Instead, there is a continuum of states closed to the magnon energies even for $J_{\mathrm{H}} \rightarrow \infty$, and they probably overlap with magnons, providing magnon decay channels, thus giving a contribution to the observed finite lifetime of magnons in inelastic neutron scattering. This also confirms the results obtained by Golosov [11]. However, no quantitative comparison between the theoretical calculations and experimental results is available yet.

Using the ferromagnetic Kondo lattice model but taking into account strong on-site correlations between $e_{g}$ electrons and AF exchange couplings among $t_{2 g}$ spins, Mancini et al [62] discovered that the competing FM, DE, and AF super-exchange interactions lead to a strong deviation of magnon dispersion close to the zone boundary from the spectrum obtained by the isotropic Heisenberg model. However, in contrast with the experimental observation [27], the calculational results indicate that magnons in the [1,1,1] direction should have the largest softening from that predicted by the nearest-neighbour Heisenberg model (see equation (7)) as compared with these in the $[1,0,0]$ and $[1,1,0]$ directions.

Both Khaliullin et al [64] and Maezono et al [65] realized the importance of the $\mathrm{e}_{\mathrm{g}}$ orbital degrees of freedom [68, 69] in the spin dynamics in FM metallic manganites. The strength of the FM interaction at a given bond strongly depends on the orbital character of $\mathrm{e}_{\mathrm{g}}$ electrons. Thus quantum fluctuations of $\mathrm{e}_{\mathrm{g}}$ orbitals are shown to strongly modulate the magnetic exchange bonds, thereby causing a renormalization of magnon dispersion. In particular, the 
short-wavelength magnons are affected because they are most sensitive to these local orbital fluctuations. This causes the unusual zone-boundary magnon softening.

Khaliullin et al [64] found that the presence of JT phonons further enhances the orbital fluctuation effect. They considered JT coupling of orbitals to the lattice, which imposes low phonon frequencies onto orbital fluctuations, thus providing the phononic contribution to the magnon self-energy. With [64] or without [65] the involvement of the JT phonons, the quantum fluctuations of the planar orbitals (i.e., $\mathrm{d}_{x^{2}-y^{2}}, \mathrm{~d}_{y^{2}-z^{2}}$, and $\mathrm{d}_{z^{2}-x^{2}}$ ) are dominant in the DE interaction, thus leading to large anisotropy of the spin dynamics. As a result, the magnon dispersion is largely renormalized in the $[1,0,0]$ and $[1,1,0]$ directions while very little affected in the $[1,1,1]$ direction [64]. The magnons along the $[1,1,1]$ direction are sensitive to all three spatial directions of exchange bonds, and therefore remain unaffected by the local symmetry breaking induced by low-dimensional orbital correlations [64, 70]. This gives an explicit explanation for the anisotropic magnon softening.

Considering the doping dependence of different orbital correlations which mediate the exchange couplings, Endoh et al [28] argued that the anomalous magnon dispersion can be described by the phenomenological Heisenberg model with extended exchange coupling constants $\left(J_{\mathrm{s}}\right)$. The theoretical results based on the local density approximation + Hubbard $U$ calculations identified the contributions to exchange coupling constants from different types of orbital states. For $\mathrm{Sm}_{0.55} \mathrm{Sr}_{0.45} \mathrm{MnO}_{3}$ with a higher doping level of $x=0.45, J_{4}$ is enhanced considerably by the $\mathrm{d}_{3 z^{2}-r^{2}}$ orbital state [28] rather than the $\mathrm{d}_{x^{2}-y^{2}}$ one for those with the lower doping level of $x=0.3$ [64]. Nevertheless, the prediction of the doping dependence of $J_{4} / J_{1}$ is not quantitatively consistent with experimental results [21] as we have mentioned in section 6.

Solovyev et al [42] argued that the zone-boundary magnon softening and the increase of $D$ with doping $(x)$ have a purely magnetic spin origin. The observed magnon softening, demonstrating the importance of the long-range FM coupling, is a natural consequence of the $\mathrm{e}_{\mathrm{g}}$-band filling in the half-metallic regime, implying that the canonical DE limit $\left(t / J_{\mathrm{H}} \rightarrow 0\right)$ is not appropriate and that neither the lattice deformation nor the orbital ordering is required for the softening. Based upon the minimal tight-binding calculation including the consideration of the super-exchange interactions between localized spins, they found that the ratio of the longerrange coupling $\left(J_{\mathrm{s}}\right)$ to $J_{1}$ including $J_{4} / J_{1}$ depends on doping $(x)$. However, the calculated doping dependence of the zone-boundary softening along the $[1,0,0]$ direction seems to be much less than experimental results (see figure 13(c)), while the agreement between the experimental and theoretical results of the magnon dispersion along the $[1,1,0]$ and $[1,1,1]$ directions is yet to be confirmed.

Magnon-phonon coupling is another candidate beyond the electronic origin to renormalize the self-energy of magnons. In many low- $T_{\mathrm{C}}$ manganites, in which unusual magnon behaviour has been observed, magneto-elastic and dynamic JT effects [71] are believed to be crucial to transport and magnetic properties. In addition, and as we described above, the observed unusual magnon behaviours, especially the magnon softening and broadening, have some relation to the JT active phonons $[23,27,44,49]$. Therefore, it is 'natural' to suggest that magnons are coupled to phonons.

Furukawa [46] has given a qualitative argument on the effects of magnon-phonon coupling for the case where their dispersions cross each other. When the interaction conserves the spin quantum number, the magnon linewidth becomes broad only when the magnon energy is higher than the magnon-phonon crossing energy. This seems to explain the observed magnon anomalous broadening close to the zone boundary. A more realistic calculation on both phonon and magnon damping was given by Woods [47]. The calculation is based upon a model Hamiltonian in which the magnon part is taken care of by the Heisenberg Hamiltonian and the magnon-phonon coupling is reproduced by the scattering of a magnon with an emission 
or absorption of a phonon. In this case the coupling manifests itself through the distortion of the lattice. By calculating the self-energy of magnons, the magnon softening and broadening have been reproduced. Due to the fact that the magnon damping is proportional to the boson population, this model explains in a natural way the enhanced damping with increasing temperature. However, the magnon-phonon coupling picture has difficulty in explaining the doping dependence of $J_{4} / J_{1}$ (see figure 13) and the insensitivity of $J_{4} / J_{1}$ to the average Asite ionic radius $(\bar{r})$ (see figure 12 ). As the phonon frequency does not vary drastically with increasing doping (for $0.25<x<0.45$ ), a large change of $J_{4} / J_{1}$ with increasing doping would not be expected. On the other hand, if phonons play a crucial role, there would be a correlation between $T_{\mathrm{C}}$ or $D$ and electron-phonon coupling strength $(g)$ [71-74,66]. While increasing $\bar{r}$ leads to rapid changes in $T_{\mathrm{C}}$, it is hard to image the insensitivity of $D$ or $J_{4} / J_{1}$ to $T_{\mathrm{C}}$ under the magnon-phonon scenario.

Finally, understanding the effect of quenched disorder on spin dynamics in FM metallic manganites is still an issue. It is known that the randomness of A-site substitution has a drastic effect on $T_{\mathrm{C}}$ as well as transport properties [39], suggesting that the disorder in the mixture of different size ions scatters the itinerant electrons and suppresses their kinetics. Motome et al [35] have studied the spin excitation spectrum in the DE model with the presence of disorder. They found that the disorder causes anomalies in the magnon spectrum including broadening, branching, and anticrossing with gap opening. The $2 k_{\mathrm{F}}$ Friedel oscillation of spin and charge density in the fully polarized FM state caused by disorder which scatters the magnons and results in anticrossing in their dispersion etc is believed to be the origin of these anomalies. According to their study, the increase of on-site disorder should enhance the magnon softening and damping. However, except the observed zone boundary broadening, which may be used to compare with the theoretical results, the insensitivity of $D$ or $J_{4} / J_{1}$ to $\sigma^{2}$ seems to rule out the possibility of on-site disorder-induced zone boundary softening (see figure 12(a)).

\section{Summary}

In this review we have described recent studies of the magnon behaviours in FM metallic manganites. We focused only on metallic perovskite manganites $\mathrm{R}_{1-x} \mathrm{~A}_{x} \mathrm{MnO}_{3}$ (e.g. $\mathrm{R}=\mathrm{La}$, $\mathrm{Nd}, \mathrm{Pr}, \mathrm{A}=\mathrm{Sr}, \mathrm{Ca}, \mathrm{Pb}$ ), although there are a lot of excellent studies of the spin dynamics in the non-metallic phase of these three-dimensional materials as well as the spin dynamics of layered manganite compounds. In spite of a great deal of experimental and theoretical effort, a clear picture of the spin dynamics including its doping and temperature dependence is yet to emerge. Theoretically, several mechanisms proposed so far have certain degrees of success accounting for anomalous magnon behaviours deviating from the simple canonical Heisenberg model. However, none of them can satisfactorily explain all of the observed results. Experimentally, considerably more work is needed to fully characterize the momentum, temperature, and doping dependence of magnons. In particular, a few important experimental measurements are essential to further test these theoretical mechanisms.

(1) The possible zone-boundary magnon softening in high- $T_{\mathrm{C}}$ manganites as compared with the simple Heisenberg model with nearest-neighbour interaction, in order to confirm whether the zone-boundary softening is a universal phenomenon in all FM metallic manganites.

(2) The full characterization of magnon dispersion in the $[1,1,1]$ direction, especially for the low- $T_{\mathrm{C}}$ manganites [27]. This requires high-energy neutrons.

(3) The systematic measurements on the doping and temperature dependence of magnon linewidth $\Gamma(\vec{q})$. 
(4) The isotope effects on magnon dispersion and damping to further test the magnon-phonon coupling.

(5) The scaling behaviour of the spin wave stiffness $D(T)$, especially the magnon non-collapse issue when $T \rightarrow T_{\mathrm{C}}$.

(6) The systematic measurement of the electronic band structure including the Fermi surfaces, which is crucial to understand the correlation effects on spin dynamics. Though progress in the study of the Fermi surface topology with high-resolution angle-resolved photoelectron spectroscopy (ARPES) has been made in the layered manganites such as $\mathrm{La}_{1.2} \mathrm{Sr}_{1.8} \mathrm{Mn}_{2} \mathrm{O}_{7}[75,76]$, it is difficult to determine the electronic structure in these three-dimensional manganites because they cannot be cleaved to obtain a reasonably good surface. Surface effects $[77,78]$ due to the surface lattice relaxation, segregation, and/or imperfection would affect the measured electronic structure by using surface sensitive techniques like ARPES.

Polarized inelastic neutron scattering will be extremely helpful for distinguishing the magnon excitations from others and extracting the truly intrinsic magnon bandwidth and linewidth, especially near the zone boundary where magnons merge with phonons. Meanwhile, the effects of lattice distortion, quenched disorder, and even phase separation should be important, especially at temperature close to $T_{\mathrm{C}}$, thus deserving further investigation. Due to the nature of the close and complex coupling between charge, lattice, orbital, and spin degrees of freedom in this class of materials, how to tailor these different interactions to reveal their effects on spin dynamics should be the main challenge.

\section{Acknowledgments}

This research was sponsored by the US Department of Energy (DOE), Basic Energy Sciences, Division of Materials Sciences and Engineering, and conducted at Oak Ridge National Laboratory (ORNL). JZ was also supported in part by the US National Science Foundation (NSF) with grant No DMR-0346826 and PD was supported in part by NSF with grant No DMR0453804. ORNL is supported by the US DOE grant No De-AC05-00OR22725 through UT/Battelle LLC.

\section{References}

[1] De Groot R A, Müller F M, van Engen P G and Buschow H J 1983 Phys. Rev. Lett. 502024

[2] Jonker G H and Van Santen J H 1950 Physica 16337 Jonker G H and Van Santen J H 1950 Physica 16599

[3] Volger J 1954 Physica 2049 Kusters R M, Singleton J, Keen D A, McGreevy R and Hayes W 1989 Physica B 155362 von Helmot R, Wecker J, Holzapfel B, Schultz L and Samwer K 1993 Phys. Rev. Lett. 712331 Jin S, Tiefel T H, McCormack M, Fastnacht R A, Ramesh R and Chen L H 1994 Science 264413

[4] Pickett W E and Singh D J 1996 Phys. Rev. B 531146

[5] Park J H, Vescovo E, Kim H-J, Kwon C, Ramesh R and Venkatesan T 1998 Nature 392794

[6] Tokura Y 1999 Colossal Magnetoresistance Oxides ed Y Tokura (London: Gordon and Breach) Kaplan T A and and Mahanti S D (ed) 1999 Physics of Manganites (New York: Kluwer Academic/Plenum) Salamon M B and Jaime M 2001 Rev. Mod. Phys. 73583

[7] Dagotto E 2003 Nanoscale Phase Separation and Colossal Magneto-Resistance: the Physics of Manganites and Related Compounds (Berlin: Springer)

[8] Zener C 1951 Phys. Rev. 81440 Zener C 1951 Phys. Rev. 82403 Zener C 1951 Phys. Rev. 83299 Goodenough J B 1955 Phys. Rev. 100564 
Anderson P W and Hasegawa H 1955 Phys. Rev. 100675 de Gennes P-G 1960 Phys. Rev. 118141

[9] Kubo K and Ohata N 1972 J. Phys. Soc. Japan 3321

[10] Furukawa N 1996 J. Phys. Soc. Japan 651174

[11] Golosov D I 2000 Phys. Rev. Lett. 843974 Golosov D I 2005 Phys. Rev. B 71014428

[12] Shannon N 2001 J. Phys.: Condens. Matter 136371 Shannnon N and Chubukov A 2002 Phys. Rev. B 65104418

[13] Rushbrooke G S et al 1974 Phase Transition and Critical Phenomena ed C Domb and M S Green (New York: Academic) equation (5.4)

[14] Squire G L 1978 Thermal Neutron Scattering (Cambridge: Cambridge University Press) Loversy S W 1984 Theory of Neutron Scattering from Condensed Matter (Oxford: Clarendon) Shirane G, Shapiro S M and Tranquada J M 2002 Neutron Scattering with a Triple-Axis Spectrometer: Basic Techniques (Cambridge: Cambridge University Press)

[15] Lowde R D 1965 J. Appl. Phys. 36884 Marshall W and Murray G 1968 J. Appl. Phys. 39380 Marshall W and Lowde R D 1968 Rep. Prog. Phys. 31705 Shirane G, Minkiewicz V J and Nathans R 1968 J. Appl. Phys. 39383

[16] Chaikin P M and Lubensky T C 1995 Principle of Condensed Matter Physics (Cambridge: Cambridge University Press)

[17] Perring T G, Aeppli G, Hayden S M, Carter S A, Remeika J P and Cheong S-W 1996 Phys. Rev. Lett. 77711

[18] Martin M C, Shirane G, Endoh Y, Hirota K, Moritomo Y and Tokura Y 1996 Phys. Rev. B 5314285

[19] Vasiliu-Doloc L, Lynn J W, Moudden A H, Arsenov A A and Shulyatev D A 1998 J. Appl. Phys. 837342 The zone boundary data for $\mathrm{La}_{0.7} \mathrm{Sr}_{0.3} \mathrm{MnO}_{3}$ are from the work of Lynn $\mathrm{J} \mathrm{W}$ et al 2006 unpublished

[20] Endoh E and Hirota K 1997 J. Phys. Soc. Japan 662264

[21] Ye F, Dai P, Fernandez-Baca J A, Sha H, Lynn J W, Kawano-Furukawa H, Tomioka Y, Tokura Y and Zhang J 2006 Phys. Rev. Lett. 96047204

[22] Urushibara A, Moritomo Y, Arima T, Asamitsu A, Kido G and Tokura Y 1995 Phys. Rev. B 5114103

[23] Moudden A H, Pinsard L, Vasiliu-Doloc L and Revcolevschi A 1996 Czech J. Physique 462163 Vasiliu-Doloc L, Lynn J W, Moudden A H, de Leon-Guevara A M and Revcolevschi A 1997 J. Appl. Phys. 815491

Vasiliu-Doloc L, Lynn J W, Moudden A H, de Leon-Guevara A M and Revcolevschi A 1998 Phys. Rev. B 5814913

[24] Hwang H Y, Dai P, Cheong S-W, Aeppli G, Tennant D A and Mook H A 1998 Phys. Rev. Lett. 801316

[25] Fernandez-Baca J A, Dai P, Hwang H Y, Kloc C and Cheong S-W 1998 Phys. Rev. Lett. 804012

[26] Lynn J W, Erwin R W, Borchers J A, Huang Q and Santoro A 1996 Phys. Rev. Lett. 764046

[27] Dai P, Hwang H Y, Zhang J, Fernandez-Baca J A, Cheong S-W, Kloc C, Tomioka Y and Tokura Y 2000 Phys. Rev. B 619553

Ye F, Dai P, Fernandez-Baca J A, Adroja D T, Perring T G, Tomioka Y and Tokura Y 2007 Preprint cond-mat/0702504

[28] Endoh Y, Hiraka H, Tomioka Y, Tokura Y, Nagaosa N and Fujiwara T 2005 Phys. Rev. Lett. 94017206

[29] Cheong S-W and Hwang H Y 1999 Colossal Magnetoresistance Oxides ed Y Tokura (London: Gordon and Breach)

[30] Tomioka Y, Kuwahara H, Asamitsu A, Kasai M and Tokura Y 1997 Appl. Phys. Lett. 703609

[31] Barilo S N, Bychkov G L, Kurnveich L A, Shiryaev S V, Kurochkin L A, Lynn J W and Vasiliu-Doloc L 2000 J. Cryst. Growth 211480

[32] Chatterji T, Regnault L P and Schmidt W 2002 Phys. Rev. B 66214408

[33] Tomioka Y, Asamitsu A, Kuwahara H, Moritomo Y and Tokura Y 1996 Phys. Rev. B 531689

[34] Fernandaz-Beca J A, Dai P, Kawano-Furukawa H, Yoshizowa H, Plummer E W, Katano S, Tomioka Y and Tokura Y 2002 Phys. Rev. B 66054434

[35] Motome Y and Furukawa N 2002 J. Phys. Soc. Japan 711419 Motome Y and Furukawa N 2003 J. Phys. Soc. Japan 72472 Motome Y and Furukawa N 2003 Phys. Rev. B 68144432 Motome Y and Furukawa N 2005 Phys. Rev. B 71014446

[36] Akahoshi D, Uchida M, Tomioka Y, Arima T, Matsui Y and Tokura Y 2003 Phys. Rev. Lett. 90177203 Mathieu R, Akahoshi D, Asamitsu A, Tomioka Y and Tokura Y 2004 Phys. Rev. Lett. 93227202

[37] Sato T J, Lynn J W and Dabrowski B 2004 Phys. Rev. Lett. 93267204

[38] Moshnyaga V, Sudheendra L, Lebedev O I, Köster S A, Gehrke K, Shapoval O, Belenchuk A, Damaschke B, van Tendeloo G and Samwer K 2006 Phys. Rev. Lett. 97107205 
[39] Rodriguez-Martinez L M and Attfield J P 1996 Phys. Rev. B 5415622

[40] Hwang H, Palstra T T M, Cheong S-W and Batlogg B 1995 Phys. Rev. Lett. 74914

[41] Radaelli P G, Iannone G, Marezio M, Hwang H Y, Cheong S-W, Jorgensen J D and Argyriou D N 1997 Phys. Rev. B 568265

[42] Solovyev I V and Terakura K 1999 Phys. Rev. Lett. 822959

[43] Kirby B J, Rhyne J J, Kaiser H, Kuwahara H and Tokura Y 2006 J. Magn. Magn. Mater. 302237

[44] Zhang J et al 2004 unpublished

[45] Harris A B 1968 Phys. Rev. 175674

[46] Furukawa N 1999 J. Phys. Soc. Japan 682522

[47] Woods L M 2001 Phys. Rev. B 65014409

[48] Reichardt W and Braden M 1999 Physica B 263416

[49] Zhang J, Dai P, Fernandez-Baca J A, Plummer E W, Tomioka Y and Tokura Y 2001 Phys. Rev. Lett. 863823

[50] Fernandez-Baca J A, Hagen M E, Dai P, Ye F, Kulda J, Tomioka Y and Tokura Y 2006 Physica B 385/386 66

[51] Dai P, Fernandez-Baca J A, Plummer E W, Tomioka Y and Tokura Y 2001 Phys. Rev. B 64224429

[52] Lynn J W, Erwin R W, Borchers J A, Santoro A, Huang Q, Peng J-L and Greene R L 1997 J. Appl. Phys. 815488

[53] Mattis D C 1981 The Theory of Magnetism (Heidelberg: Springer)

[54] Collins M 1989 Magnetic Critical Scattering (Oxford: Clarendon) p 29

[55] Adams C P, Lynn J W, Mokovskii Y M, Arsenov A A and Shulyatev D A 2000 Phys. Rev. Lett. 853954

[56] Lynn J W 2000 J. Supercond.: Incorporating Novel Magnetism 13263

[57] Hennion M, Moussa F, Biotteau G, Rodríguez-Carvajal, Pinsard L and Revcolevschi A 1998 Phys. Rev. Lett. 811957

Hennion M, Moussa F, Lehouelleur P, Wang F, Ivanov A, Mukovskii Y M and Shulyatev D 2005 Phys. Rev. Lett. 94057006

[58] Dagotto E, Hotta T and Moreo A 2001 Phys Rep. 3441

Dagotto E 2005 New J. Phys. 767

Dagotto E 2005 Science 309257

[59] Dai P, Fernandez-Baca J A, Wakabayashi N, Plummer E W, Tomioka Y and Tokura Y 2000 Phys. Rev. Lett. 852553

[60] Feng S-S and Mochena M 2005 J. Phys.: Condens. Matter 173895

[61] Kapetanakis M D, Manousaki A and Perakis I E 2006 Phys. Rev. B 73174424

[62] Mancini F, Perkins N B and Plakida N M 2001 Phys. Lett A 284286

[63] Kaplan T A, Mahanti S D and Su Y-S 2001 Phys. Rev. Lett. 863634

[64] Khaliullin G and Kilian R 2000 Phys. Rev. B 613494

[65] Maezono R, Ishihara S and Nagaosa N 1998 Phys. Rev. B 57 R13993 Maezono R and Nagaosa N 2000 Phys. Rev. B 611189

[66] Edwards D M 2002 Adv. Phys. 511259 and references therein

[67] Wurth P and Müller-Hartmann E 1998 Eur. Phys. J. B 5403

[68] Kugel K and Khomskii D I 1973 Sov. Phys._JETP 37725

[69] Ishihara S, Yamanaka M and Nagaosa N 1997 Phys. Rev. B 56686

[70] Krivenko S, Yaresko A, Khaliullin G and Fehke H 2004 J. Magn. Magn. Mater. 272/276 458

[71] Millis A J, Littlewood P B and Shrainman B I 1995 Phys. Rev. Lett. 745144 Millis A J, Shrainman B I and Mueller R 1996 Phys. Rev. Lett. 77175

[72] Röder H, Zang J and Bishop A R 1996 Phys. Rev. Lett. 761356 Zang J, Bishop A R and Röder H 1996 Phys. Rev. B 54 R8840

[73] Millis A J, Mueller R and Shrainman B I 1996 Phys. Rev. B 545389 Millis A J, Mueller R and Shrainman B I 1996 Phys. Rev. B 545405

[74] Green A C 2001 Phys. Rev. B 63205110 Hohenadler M and Edwards D M 2002 J. Phys.: Condens. Matter 142547

[75] Chuang Y D, Gromko A D, Dessau D S, Kimura T and Tokura Y 2001 Science 2921509

[76] Mannella N, Yang W L, Zhou X J, Zheng H, Mitchell J F, Zaanen J, Devereaux T P, Nagaosa N, Hussiain Z and Shen Z X 2005 Nature 438474

Sun Z, Chuang Y-D, Fedorov A V, Douglas J F, Reznik D, Weber F, Aliouane N, Argyriou D N, Zheng H, Mitchell J F, Kimura T, Tokura Y, Revcolevschi A and Dessau D S 2006 Phys. Rev. Lett. 97056401

[77] Choi J, Zhang J, Liou S-H, Dowben P A and Plummer E W 1999 Phys. Rev. B 5913453 Dulli H, Dowben P A, Liou S-H and Plummer E W 2000 Phys. Rev. B 77570

[78] Freeland J W, Gray K E, Ozyuzer L, Berghuis P, Badica E, Kavich J, Zheng H and Mitchell J F 2004 Nat. Mater. 462 